\title{
Mitochondrial membrane proteins and VPS35 orchestrate selective removal of mtDNA.
}

David Pla-Martin ( $\square$ dplamart@uni-koeln.de )

University of Cologne https://orcid.org/0000-0002-5189-0723

Ayesha Sen

Uniklinik Köln

Sebastian Kallabis

University of Cologne

Julian Nüchel

Max Plank Institut Ageing

Kanjanamas Maliphol

Uniklinik Köln

Julia Hofmann

Uniklinik Köln

Markus Krüger

University of Cologne

Rudolf Wiesner

University of Cologne https://orcid.org/0000-0003-1677-4476

\section{Article}

Keywords: mitochondrial DNA, Twinkle, VPS35

Posted Date: August 9th, 2021

DOl: https://doi.org/10.21203/rs.3.rs-629802/v1

License: (c) (i) This work is licensed under a Creative Commons Attribution 4.0 International License.

Read Full License

Version of Record: A version of this preprint was published at Nature Communications on November 7th, 2022. See the published version at https://doi.org/10.1038/s41467-022-34205-9. 
1 Mitochondrial membrane proteins and VPS35 orchestrate selective removal of mtDNA.

2 David Pla-Martin ${ }^{1,2^{*}}$, Ayesha Sen ${ }^{1}$, Sebastian Kallabis ${ }^{2}$, Julian Nüchel ${ }^{3}$, Kanjanamas Maliphol ${ }^{1}$, 3 Julia Hofmann ${ }^{1}$, Marcus Krüger ${ }^{2}$ and Rudolf J. Wiesner ${ }^{1,2,4}$

5 Affiliations

$6 \quad{ }^{1}$ Center for Physiology and Pathophysiology, Institute of Vegetative Physiology, University of

7 Köln, Köln, Germany

$8{ }^{2}$ Cologne Excellence Cluster on Cellular Stress Responses in Aging-associated Diseases

9 (CECAD), University of Köln, Köln, Germany

$10{ }^{3}$ Center for Biochemistry, Faculty of Medicine, University of Köln, Köln, Germany

$11{ }^{4}$ Center for Molecular Medicine Cologne, University of Köln, Köln, Germany

12 *Corresponding author: David Pla-Martin, PhD; dplamart@uni-koeln.de

\section{Abstract}

Integrity of mitochondrial DNA (mtDNA), encoding several subunits of the respiratory chain, is essential to maintain mitochondrial fitness. Mitochondria, as a central hub for metabolism, are affected in a wide variety of human diseases but also during normal ageing, where mtDNA integrity is compromised. Mitochondrial quality control mechanisms work at different levels, and mitophagy and its variants are critical to remove dysfunctional mitochondria together with mtDNA to maintain cellular homeostasis. Understanding the mechanisms governing a selective turnover of mutation-bearing mtDNA without affecting the entire mitochondrial pool is fundamental to design therapeutic strategies against mtDNA diseases and ageing. Here we show that mtDNA depletion after expressing a dominant negative version of the mitochondrial helicase Twinkle, or by chemical means, is due to an exacerbated mtDNA turnover. Targeting of nucleoids is controlled by Twinkle which, together with the mitochondrial transmembrane proteins ATAD3 and SAMM50, orchestrate mitochondrial membrane remodeling to form extrusions. mtDNA removal depends on autophagy and requires the vesicular trafficking protein VPS35 which binds to Twinkle-enriched mitochondrial subcompartments upon mtDNA damage. Stimulation of autophagy by rapamycin selectively removes mtDNA deletions which accumulated during muscle regeneration in vivo, but without affecting mtDNA copy number. With these results we unveil a new complex mechanism specifically targeting and removing mutant mtDNA which occurs outside the mitochondrial network. We reveal the molecular targets involved in a process with multiple potential benefits against human mtDNA related diseases, either inherited, acquired or due to normal ageing. 
39 The accumulation over time of mutations in the mitochondrial genome (mtDNA) is a common 40 process which has been shown to occur in many tissues ${ }^{1}$ and is probably one of the hallmarks 41 of aging ${ }^{2}$. MtDNA is present in thousands of copies per cell, hence, impairment of mitochondrial 42 function is observed only when the percentage of mutated mtDNA molecules surpasses a specific 43 threshold ${ }^{3}$.

44 Cells possess a plethora of quality control mechanisms to survey the intactness of DNA, RNA 45 and proteins, but also of entire organelles. In addition to bulk autophagy, which is responsible for 46 the continuous and non-selective turnover of cellular material activated during nutrient shortage, 47 specific mechanisms are initiated to remove malfunctioning organelles upon damage. The 48 process of mitophagy has been investigated extensively in recent years as an important salvage 49 pathway to remove dysfunctional mitochondria ${ }^{4}$. The ongoing fission and fusion events of the 50 dynamic mitochondrial network are important processes in order to survey mitochondrial quality 51 by predisposing those parts of the network with impaired function to degradation. Mitochondrial 52 fission is a requirement to initiate mitophagy of damaged mitochondria ${ }^{4}$, whereas knockout of 53 key players of mitochondrial fusion has been shown to induce mtDNA instability, either by causing 54 a rapid accumulation of mtDNA alterations over time ${ }^{5}$ or by mtDNA depletion ${ }^{6}$.

55 Recently, a process with a high level of specificity involving mitochondrial derived vesicles (MDVs) 56 was shown to remove not the complete organelle, but rather mitochondrial fragments containing 57 specific cargo ${ }^{7,8}$. This mechanism requires the coordination of mitochondrial dynamics, mitophagy 58 and also the vacuolar protein sorting (VPS) or retromer complex. In this process, changes in the mitochondrial membrane potential and the oxidation state of mitochondrial subcompartments induce the curvature of the membrane which is followed by recruitment of PINK1 and PARKIN ${ }^{9}$. The retromer complex, formed by VPS26, VPS29 and VPS35, provides the force to generate a vesicle which is then delivered to lysosomes or peroxisomes in a process which is independent of the autophagy proteins ATG5 or LC3 ${ }^{10-12}$. In summary, mitophagy and its variants are crucial pathways to degrade parts of the mitochondrial network, thus maintaining cellular fitness. Many inherited forms of neurodegenerative diseases are examples for insufficient mitochondrial quality control. Mutations of specific receptors involved in targeting dysfunctional organelles to mitophagy like PINK1 and PARKIN, but also malfunction of lysosomal proteins ATP13A2 and LAMP3 among many others, as well as mutations in the retromer component VPS $35^{13}$ cause familial forms of Parkinson's disease. Parkinson's disease is caused by the specific degeneration of dopaminergic neurons which have been comprehensively shown to be a hotspot for the accumulation of large scale mtDNA deletions during normal aging ${ }^{14,15}$ making mitochondrial quality control mechanisms especially important in these cells. Unfortunately, these mechanisms are not sufficient to counteract the often progressive phenotype of patients suffering from PD and other diseases caused by either maternally inherited or by acquired mtDNA alterations, the latter due to mutations in proteins essential for mtDNA replication and maintenance ${ }^{16}$. Thus, therapeutic approaches to increase mitochondrial quality control and counteract the progression 
undesirable effects are still a hallmark ${ }^{19}$. Therefore, understanding the specific mechanisms governing mtDNA turnover is pivotal to develop new therapeutic strategies against these syndromes.

Expressing the mitochondrial helicase Twinkle bearing dominant negative mutations causing mitochondrial disease in patients induces mtDNA instability ${ }^{20}$. Expression of the disease related PEO1 Twinkle mutation K320E (from now on K320E) in mouse models accelerates the accumulation of mtDNA deletions in postmitotic tissues like heart and muscle ${ }^{21,22}$ during aging and induce mtDNA depletion in proliferating cells such as epidermis and cartilage ${ }^{23,24}$. Recently, it has been shown that muscles from those mice accumulated deletions, but the main alterations were gene duplications, leading to a vast variety of different enlarged and rearranged molecules ${ }^{25}$. Using a combination of in vivo and in vitro approaches, we have now identified the proteins involved in a new mechanism for targeting and specific degradation of mtDNA to avoid the accumulation of such mutations. Expression of K320E induces the formation of mitochondrial extrusions, much larger than MDVs, which are consecutively engulfed by autophagosomes and fused with lysosomes. Elimination of altered mtDNA molecules is preceded by the relocation of nucleoids to the poles of mitochondria, a process controlled by the interaction between the mitochondrial inner membrane protein ATAD3 and the nucleoid protein Twinkle. The translocase protein SAMM50 and VPS35 are essential to provide the required selectivity and specificity for $m t D N A$ elimination. We show that stimulation of autophagy by rapamycin in vivo is sufficient to specifically remove deleted mtDNA, but without affecting the total mtDNA copy number. Thus, modulation of autophagy in vivo can be used as an approach to counteract the accumulation of mutations in mtDNA observed in several mitochondrial pathologies and during aging.

\section{RESULTS}

\section{Accumulation of mtDNA mutations in skeletal muscles in vivo does not induce mitophagy} In a previous work we have found that, in extraocular muscles, which are first affected in Peo1 patients, mtDNA alterations preferentially accumulated in fast twitch in contrast to slow oxidative fibers, indicating important differences in mitochondrial quality control mechanisms in different muscle fiber types ${ }^{22}$. To study the nature of these mechanisms surveillant mtDNA integrity, we first analysed fast-twitch M. tibialis anterior (TA) and slow-oxidative M. soleus (SOL), muscles both rich in fibers with a high mitochondrial content in mice, but with a preferentially glycolytic (TA) vs. oxidative metabolism (SOL), respectively. As shown before by deep sequencing ${ }^{25}$, both muscles showed an accumulation of many mtDNA alterations in 24 months old animals (Fig. 1a). K320E mutant mice carry a wide variety of reorganized mtDNA molecules ${ }^{25}$, causing an inefficient amplification reaction leading to a smear of products, however there were no changes in total mtDNA copy number (Fig. 1b). Noteworthy, TA from aged wt mice also showed many mtDNA alterations, while only few were found in SOL. By conventional PCR, we analysed the presence of deletions common in aged mice ${ }^{22}$ and selected a deletion covering about 4000bp (Mus musculus mtDNA- ${ }^{983-4977}$ ), which was present in both mutant muscles (Fig. S1a). Considering 
that mtDNA copy number is on average $20 \%$ higher in SOL than in TA (Fig. S1b), we performed

119 qPCR quantification using the D-Loop region as a reference and found that indeed, this deletion was on average 20 times more abundant in TA compared to SOL (Fig. 1C).

121 Steady state levels of common mitochondrial autophagy markers showed that in SOL of K320E ${ }^{\text {skm }}$ mice, the general autophagy adaptors p62 and LC3 were significantly decreased while levels were similar in TA (Fig. 1d, e). Levels of the specific mitophagy adaptor Optineurin (OPT) and the mitochondrial marker TOM20 were similar in all samples. In situ immunofluorescence of LC3 and p62 confirmed reduced puncta of those proteins (Fig. S1c-f). Since such a reduction could reflect both an increase or a decrease of autophagy flux, we blocked autophagy flux by chloroquine, and analysed the conversion of LC3-I to LC3-II (Fig. 1f, g). We did not observe an increased autophagy flux in K320E mice compared to control mice. However, in SOL for K320E mice, chloroquine was not inducing a change in the LC3 ratio suggesting that, in this muscle, autophagy flux was already at maximum level in steady state.

We therefore hypothesized that oxidative fibres, which are most dependent on intact mitochondria, possess a faster mitochondrial turnover, hence maintaining mutated mtDNA molecules more efficiently below a pathogenic threshold. To visualize protein synthesis in vivo, we fed our mice with heavy lysine $\left({ }^{13} \mathrm{C}_{6}\right.$-lysine $)$ for two weeks in order to get an estimate for mitochondrial turnover rates ${ }^{26}$. Here, we selected SOL and M. extensor digitorum longus (EDL) for analysis, since the latter contains almost exclusively glycolytic fibers. We first confirmed that heavy Lysine incorporation was equal in all animals (Fig. S2a, b). We analysed $\mathrm{H} / \mathrm{L}$ (heavy/light) ratios of detected proteins (1286 and 1224 for SOL, 1042 and 1018 for EDL in wild-type and $\mathrm{K}_{320 \mathrm{E}^{\mathrm{skm}}}$ mice, respectively) and filtered the mitochondrial proteins using Mitocarta 3.0 (Supplementary Table 1; Fig. S1c). Surprisingly, no mitochondrial protein was showing differential incorporation of heavy lysine comparing K320E mutant and wild-type animals, neither in EDL nor in SOL, suggesting that bulk mitochondrial turnover, here measured as an increase of mitochondrial biogenesis, is not enhanced in muscles bearing mtDNA alterations (Fig. 1h).

\section{Autophagy is required for depletion of mtDNA following oxidative damage}

146 To further dissect the molecular pathways activated upon mtDNA instability, we generated stable

147 C2C12 myoblast cell lines expressing tagged versions of Twinkle and K320E. Colocalization of 148 these variants with mitochondria (outer membrane marker TOM20, Fig. S3a, b) as well as 149 colocalization with mtDNA and the mtDNA binding protein TFAM was confirmed (Fig. S3c, d), 150 showing that Twinkle is enriched in mitochondrial nucleoids.

151 To investigate if K320E expression leads to activation of autophagy or mitophagy in these cells, 152 C2C12 myoblasts were additionally transfected with plasmids encoding LC3-GFP-mCherry or 153 Fis1p-GFP-mCherry. Expression of K320E induced the accumulation of autolysosomes marked 154 by LC3-GFP-mCherry (Fig. 2a, b; magenta signal). In agreement with our in vivo pSILAC results, 155 expression of Fis1p-GFP-mCherry, a mitophagy reporter, showed no activation of acute 156 mitophagy (Fig. 2c, d). Previous studies have demonstrated that the in vitro expression of several 157 Twinkle missense mutations often leads to accumulation of mtDNA replication intermediates, 
producing mtDNA depletion ${ }^{27}$. Consistently, expression of K320E leads to mtDNA depletion (Fig. $2 e$ ), but this was not related to a decrease in mtDNA replication rate, as observed by analysis of mtDNA replication foci in BrdU labelled cells (Fig. S4a, b). We hypothesized that accumulation of lysosomes and mtDNA depletion after expressing K320E was reflecting a novel selective mtDNA degradation process. Thus, we blocked lysosomal activity using chloroquine and showed that, indeed, under these conditions, mtDNA levels remained at control levels (Fig. 2f). Finally, we analysed the spatial relationship between Twinkle foci and autophagy structures, using LC3 as an autophagosome marker and Lamp1 as a late endosomal marker, respectively. As expected, most wt Twinkle foci distributed in a pattern reflecting the mitochondrial network and were independent of autophagy markers (Fig. 2g-j). However, colocalization with LC3 and LAMP1, respectively, was observed with $\mathrm{K} 320 \mathrm{E}$.

The increased mtDNA degradation flux in K320E cells suggests that expression of this missense mutation harms mtDNA by unknown means. To visualize in situ mtDNA damage, we searched for the presence of 8-hydroxy-2'deoxyguanosine (8-OHdG), a specific base modification induced

172 by reactive oxygen species ${ }^{28}$. We could not observe any specific staining with $\alpha-8-O H d G$ in 173 steady state levels (Fig. S4c, d), however when cells were incubated in presence of chloroquine, 174 we detected a specific accumulation of 8-OHdG decorating the mitochondrial network only in 175 K320E expressing cells (Fig. 2k, I). In summary, our data indicate that K320E induces mtDNA 176 depletion in a lysosome-dependent manner without involving acute mitophagy, pointing to a more 177 specialized path.

178 To further analyse the contribution of autophagy to mtDNA turnover, we turned to Atg5 KO MEFs 179 and expressed Twinkle variants (Fig. 3a), since Atg5 has been shown to be important in quality 180 control after mitochondrial damage ${ }^{29}$. Analysis of mitochondrial nucleoids showed that K320E 181 expression decreased nucleoid foci number in Atg5 wt cells (Fig. 3b). In contrast, in Atg5 KO 182 cells, foci number was not altered by K320E expression (Fig. 3c). Nevertheless, expression of 183 K320E reduced mtDNA copy number in both Atg5 WT and Atg5 KO cells (Fig. 3d, e). Analysis of 184 mtDNA replication by BrdU labelling showed that, in contrast to wt cells, expression of K320E in 185 autophagy deficient cells led to a reduced number of foci replicating mtDNA within the 186 mitochondrial network (Fig. 3f, g). Chloroquine treatment also restored mtDNA copy number in 187 Atg5 WT cells expressing K320E but not in Atg5 KO cells (Fig. 3h). Our data suggest that in 188 contrast to wt cells where K320E induces mtDNA depletion linked to an increased nucleoid 189 turnover rate, in autophagy deficient cells, mtDNA depletion is caused by reduced mtDNA 190 replication, presumably to avoid accumulation of excessive mtDNA damage.

191 Therefore, to evaluate the role of autophagy in mtDNA depletion, we induced mtDNA alterations 192 by growing these cells for 7 days in medium containing Ethidium Bromide (EtBr), which we also 193 found to provoke the accumulation of 8-OHdG within the mitochondrial network (Fig. S4c, e). 194 Morphological analysis of mitochondrial nucleoids showed that EtBr decreases foci number in 195 Atg5 WT cells but not in Atg5 KO (Fig. 3i-k). In line with this, Atg5 control cells showed consistent 196 mtDNA depletion, whereas in Atg5 KO cells mtDNA copy number remained unchanged (Fig. 3l). 
This EtBr-induced depletion could not be recovered by overexpression of wt Twinkle, indicating again a prominent role of autophagy in mtDNA depletion in this case.

199 All these data together confirm that mtDNA damage induces activation of a mtDNA turnover mechanism, which is dependent on autophagosome formation and specifically degrades mtDNA but it does not activate an acute mitophagy response.

202

mtDNA instability induces the formation of mitochondrial protrusions prior to lysosomal degradation

205 One of the most surprising features we noticed while studying cellular localization of Twinkle was that TOM20, a mitochondrial outer membrane marker, does not consistently overlap with Twinkle, a mitochondrial matrix protein (Fig. 4a and S3a, b). Live imaging microscopy using $\mathrm{C} 2 \mathrm{C} 12$ clones expressing Twinkle-mCherry showed that occasionally, Twinkle moved to Mitotracker Greennegative poles of the network releasing a small particle which was also Mitotracker-negative (Fig. $4 \mathrm{~b}$, arrows). These particles were more evident in K320E expressing cells, where most of K320E was residing in such Mitotracker-negative structures (Fig. 4c). As Mitotracker green is independent of the inner membrane potential, we ruled out the possibility that these regions were depolarized areas of the mitochondrial network but mitochondrial regions with different cardiolipin 214 composition.

215 To determine Twinkle localisation with high resolution, we used Twinkle-APEX2 fusion versions 216 in $\mathrm{C} 2 \mathrm{C} 12$ cell lines. APEX2 generates a black precipitate in the presence of DAB, which is visible 217 by electron microscopy. Twinkle-APEX2 distribution in the matrix was heterogeneous and the 218 organization of mitochondria and other cellular organelles was undisturbed by its expression (Fig. 219 4d). K320E-APEX2, however, accumulated in poles of the network, sometimes close to abnormal 220 cristae structures (Fig. 3d, red arrows) and also inside lysosomes (Fig. 4d, red stars). Such multi221 membrane structures were also observed in cells expressing a mitochondrial matrix targeted 222 APEX2, but in that case, they did not show DAB precipitate (Fig. 4d, red stars). A very recent 223 study demonstrated that mitochondrial fission occurring at mitochondrial poles is followed by 224 mitophagy of mitochondrial compartments containing non-replicative mtDNA, and that this is preceded by mitochondrial cristae reorganization ${ }^{30}$. Indeed, electron tomography of K320E expressing cells revealed that these structures are derived from the inner mitochondrial membrane and that they are formed by protrusions containing reorganized mitochondrial cristae (Fig. 4e).

Twinkle interacts with membrane proteins to facilitate mtDNA removal

We speculated that mtDNA targeting prior to degradation is controlled by specific protein-protein interactions. Thus, as Twinkle follows mtDNA positioning and degradation through lysosomes, we sought to investigate how nucleoids containing Twinkle distribute in the mitochondrial matrix by analysing the Twinkle interactome. We performed immunoprecipitation of wt Twinkle fused to a V5-APEX2 epitope followed by mass spectrometry analysis. As expected, the majority of proteins interacting with Twinkle were related to mtDNA replication, transcription and translation 
(Supplementary Table 2), but only a few interactions were found to be significant (Fig. 5a, blue dots), one of them being ATAD3, an AAA-ATPase previously linked to anchoring and distribution of mtDNA to the inner membrane ${ }^{31}$. Direct co-immunoprecipitation and immunofluorescence confirmed the physical interaction between ATAD3 and Twinkle (Fig. 5b; Fig. S5a) enlightening Twinkle as an important part of the link between mitochondrial nucleoids and the inner membrane.

242 To evaluate the role of these proteins in mtDNA turnover, we generated constitutive Atad3 knockdown (KD) clones (shAtad3_1+2, shAtad3_3; Fig. S5b, c). Atad3 KD clones showed steady state levels of mtDNA copy number comparable to control lines (Fig. $5 c, d$ ) and no changes in mitochondrial morphology (Fig. S5d). After one week of mild treatment with $\mathrm{EtBr}$, control cells showed a persistent decrease of mtDNA copy number. However, this depletion was not observed in Atad3 KD clones (Fig. 5d). Number of nucleoid foci per cell was also higher in downregulated clones than in control cells upon EtBr treatment (Fig. 5e). These data suggest that nucleoid binding to the mitochondrial inner membrane through a Twinkle-ATAD3 interaction is essential for mtDNA elimination upon mtDNA damage.

mtDNA turnover requires coordination of the retromer complex in a process independent of MDVs

254 We initially hypothesized that selective removal of mitochondrial fragments containing mtDNA was carried out through MDVs ${ }^{7}$. The E3 Ubiquitin ligase MAPL has been shown to direct mitochondrial cargo to peroxisomes ${ }^{32}$, while the endosomal adaptor Tollip specifically divert MDVs to lysosomes ${ }^{8}$. In both cases, the VPS35-retromer complex initiates the force to generate a vesicle ${ }^{10}$. Thus, in order to investigate if the specific mtDNA degradation we observed follows the MDV or a new pathway, we studied VPS35 and MAPL localization in Twinkle-mCherry expressing cells. Consistent with previous studies, expression of MAPL-GFP induced mitochondrial fission and formation of structures excluding TOM20 (Fig. S6a) ${ }^{32}$. However, Twinkle was also excluded from those particles. Chloroquine treatment for $4 \mathrm{~h}$ was sufficient to induce a rapid accumulation of Twinkle positive structures excluding TOM20 and MAPL, suggesting that the fate of those particles is the lysosomal compartment (Fig. S5b). In addition, we also corroborate that Twinkle colocalizes with VPS35 but excludes MAPL (Fig. S6c, d). Next, we treated these cells as well with EtBr to induce mtDNA damage. One week of treatment was sufficient to reduce mtDNA copy number (Fig. S7a). Image quantification revealed that the percentage of VPS35 containing Twinkle was strongly increased upon EtBr treatment (Fig. 6a-c), even when the overall steady state levels of VPS35 structures did not change (Fig. 6d). Importantly, VPS35 also colocalized with dsDNA (Fig. 6e).

271 We then generated Vps35 KO clones in MEFs and selected two monoclonal lines targeting exon

2724 (Vps35_Ex4) and exon 5 (Vps35_Ex5) (Fig. S6b, c). Vps35 KO cells showed no changes in 273 mtDNA foci number but reduced levels of mtDNA copy number in steady state (Fig. 6f-h) and 274 mitochondrial fragmentation (Fig. S7d). After 7 days of EtBr treatment, the number of mtDNA foci was further decreased (Fig. S7e, f) and mtDNA depletion was enhanced (Fig. S7g, h). We analysed the activation of canonical mitophagy in steady state by expressing Fis $1 p$-GFP-mCherry 
(Fig. 6i) and found that, indeed, in Vps35 KO cells, acute mitophagy was activated (Fig. 6j).

278 Moreover, VPS35 protein level was shown to be affected by lysosomal function, as chloroquine 279 induced VPS35 accumulation in K320E cells (Fig. S7i, j) without interfering with late endosomes 280 or mitochondrial content (Fig. S7i-n).

281 We hypothesize that VPS35 is necessary to fine-tune the elimination of mutated mtDNA without 282 activating acute mitophagy and sought to investigate how VPS35 is recruited to mitochondria upon mtDNA damage. Thus, we performed VPS35 IP followed by MS analysis under basal conditions and in cells treated 7 days with $\mathrm{EtBr}$ (Fig. S8a). As expected, in basal medium the protein profile of VPS35 IP revealed poor interaction with mitochondrial proteins (Fig. 7a, red dots). Upon $\mathrm{EtBr}$ treatment, however, the association of mitochondrial proteins was markedly increased (Fig. 7b, c). Among them, we noticed the presence of mitochondrial membrane proteins such as VDAC1 and VDAC3, TIMMDC1 or TOMM40 as well as SAMM50, which interaction was confirmed by coimmunoprecipitation (Fig. 7d). The comparison of IP protein profiles of cells in basal vs. EtBr medium highlighted also a strong enrichment of the mAAA protease SPG7 (Fig. S8b). Remarkably, comparison between the IP protein profiles from Twinkle and K320E revealed also a strong enrichment of SAMM50 upon K320E immunoprecipitation (Fig. 7e). SAMM50 belongs to the sorting and assembly machinery in the mitochondrial intermembrane space and has important functions in the biogenesis of respiratory complexes, cristae morphology and mitochondrial shape ${ }^{33}$. It was also described as a regulator of PINK1-Parkin mediated mitophagy but excluding the mtDNA for degradation ${ }^{34}$. Immunoprecipitation of Twinkle confirms that, after mtDNA damage with EtBr, Twinkle can physically interact with SAMM50 (Fig. 7f). Thus, we hypothesize that SAMM50, a protein located in the mitochondrial outer membrane, could serve as a platform to recruit VPS 35 and eliminate mitochondrial subcompartments containing damaged mtDNA. Consequently, we generated SAMM50 shRNA KD clones and expressed Twinkle-mCherry (Fig. 7g; Fig. S8c, d). EtBr does not affect the overall population of VPS35 but enhances recruitment to Twinkle (Fig. 7g-i). However, the percentage of VPS35Twinkle contacts were reduced in absence of SAMM50 (Fig. 7i). To decipher if VPS35 recruitment shows Twinkle specificity, we examined VPS35 colocalization with LRPPRC, a native mitochondrial matrix protein (Fig. S8e). Again, the overall number of VPS35 particles was unchanged by EtBr treatment (Fig. S8f) and in this case, we observed that VPS35 contacts with LRPPRC were not increased and therefore, there was no effect following Samm50 downregulation (Fig. S8g).

309 In summary, our data thus demonstrate that VPS35 is required for mitochondrial quality control 310 in the presence of mtDNA alterations. We show that a mechanism to specifically remove mtDNA 311 is activated when mtDNA damage is induced both genetically or chemically by $\mathrm{K} 320 \mathrm{E}$ or by $\mathrm{EtBr}$, 312 respectively. ATAD3 is required for specific membrane localization of nucleoids, SAMM50 confers 313 nucleoid specificity and VPS35 is involved in fine-tuning of this selective process, removing 314 specific parts of mitochondria containing affected nucleoids, thus avoiding the activation of acute mitophagy which would affect the functional mitochondrial pool.

\section{Rapamycin eliminates mtDNA alterations without affecting copy number}


Since our data highlight that autophagy, together with a specific nucleoid extraction mechanism,

319 plays an important role in maintaining mtDNA fitness in vitro, we aimed to test whether this is also

320 relevant in vivo. We have previously shown that expression of K320E in skeletal muscle leads to

321 the accumulation of mtDNA alterations, unfortunately only in very old animals, making this model

322 not very convenient ${ }^{22}$ (Fig. 1). However, expression of K320E in muscle satellite cells (Pax7-

$323 \mathrm{Cre}{ }^{\mathrm{ERT}} ; \mathrm{K}_{2} 20 \mathrm{E}^{\mathrm{msc}}$ ), followed by cardiotoxin-induced muscle damage and one week of

324 regeneration, shows a rapid accumulation of mtDNA alterations, leading to newly generated,

325 cytochrome-c-oxidase (COX) negative fibers (stained blue, Fig. S8a, b), while mtDNA copy

326 number remained stable (Fig. S8c). In several mitochondrial disease models, it has been shown

327 that rapamycin, an activator of autophagy through the specific inhibition of mTORC1, is able to

328 revert mitochondrial dysfunction and ameliorate disease progression. This probably occurs by

329 stimulating mitochondrial turnover mechanisms ${ }^{35}$, and, in vitro it was also shown to direct

330 selection against mtDNA mutations ${ }^{36}$. Interestingly, genetic induction of autophagy was shown

331 to reduce mtDNA deletions in Drosophila ${ }^{37}$. Thus, to test if activation of autophagy can purify

332 mtDNA alterations also in mammals in vivo, we used this muscle regeneration paradigm in

333 combination with rapamycin treatment. Regenerated muscles from vehicle treated $\mathrm{K}_{320 \mathrm{E}^{\mathrm{msc}}}$ mice

334 showed a prominent accumulation of COX negative fibers indicating mitochondrial dysfunction

335 (Fig. 8a). In contrast, mice treated with rapamycin showed much less COX deficient cells in the

336 regenerated area (Fig. 8a-c). Consistently, qPCR analysis revealed that mtDNA copy number

337 remained unchanged (Fig. 8d), while mtDNA alterations were absent and thus had been purified

338 (Fig. 8e). We noticed that COX staining was much lighter in rapamycin treated animals,

339 suggesting a change in mitochondrial OXPHOS activity. Vehicle treated mice showed a

340 predominant accumulation of glycolytic fiber type $2 b$ in both wt and K320E ${ }^{\mathrm{msc}}$ mice (Fig. S9d),

341 while in rapamycin treated wt mice, fiber type staining showed a predominant shift towards

342 mitochondria rich type I fibers (Fig. S9e). Interestingly, in $\mathrm{K}_{2} 20 \mathrm{E}^{\mathrm{msc}}$ mice regenerated fibers

343 showed a mixed myosin heavy chain pattern after one week of regeneration. These data indicate

344 that rapamycin can be used as a modulator of mitochondrial turnover which specifically purifies

345 mutated mtDNA species, thus ameliorating mitochondrial dysfunction, albeit changing muscle

346 fiber type composition.

347

348 Discussion

349 Autophagy and specifically mitophagy and its variants are well-established pathways for

350 mitochondrial turnover, which is essential to maintain mitochondrial fitness ${ }^{4}$. Loss of

351 mitochondrial quality control mechanisms, either by specific mutations of key players or by

352 reduced autophagy activity, strikingly correlates with the acquirement of mtDNA mutations ${ }^{38}$.

353 However, an exacerbated activation of mitophagy may lead to a serious reduction of the 354 mitochondrial pool, thus affecting cellular energy supply ${ }^{39}$. Therefore, the fine-tuned regulation

355 of mitochondrial quality control mechanisms is essential to maintain cellular energy homeostasis.

356 Although toxic substances can cause mitochondrial damage, the most prevalent reason is the

357 accumulation of alterations in mtDNA due to replication errors. Mutations in genes encoding 
proteins involved in mtDNA replication, like the helicase Twinkle, and in mtDNA maintenance cause mutations leading to mitochondrial diseases, with brain and skeletal muscle being regularly affected. In addition, somatic mutations in mtDNA accumulate during the normal aging process in many organs in humans ${ }^{3}$, leading to a tissue mosaic where few cells with mitochondrial dysfunction, caused by high mutation loads, are embedded in normal tissue ${ }^{40}$.

363 In general, tissues most depending on mitochondrial function are most severely affected when 364 carrying mtDNA mutations. Paradoxically, we found that expression of the dominant negative 365 K320E mutation of Twinkle in extraocular muscle shows a remarkable differential vulnerability of muscle fiber types, with mitochondrial dysfunction especially affecting fibers with a glycolytic metabolism ${ }^{22}$. In agreement with these results, we found less mtDNA alterations in aged SOL, a muscle rich in type I fibers which mostly rely on mitochondrial ATP production, compared to the TA mostly composed of fast twitch, glycolytic fibers (Fig. 1). Noteworthy, different muscles rich in oxidative vs. glycolytic fibers show notable differences in the expression of genes involved in mitochondrial dynamics ${ }^{41}$, making oxidative muscles more resistant to ageing related dysfunction 42. In fact, our data shows that SOL expressing K320E already has an increased flux in steady state but this was not related to increased mitochondrial turnover. Nevertheless, mitophagy, understood as the specific removal of the entire damaged organelle, does not provide the required selectivity to remove only mutated mtDNA. Hence, the existence of a specific turnover mechanism has been postulated, but not been proven yet ${ }^{43}$.

In contrast to terminally differentiated muscle of aged mice, proliferating cells in culture did not accumulate mtDNA alterations upon expression of K320E, instead this led to mtDNA depletion. Both K320E expression or mild treatment with $\mathrm{EtBr}$ induced the accumulation of mtDNA damage. $\mathrm{EtBr}$ intercalates between base pairs and slows down mtDNA replication. It is accepted that $\mathrm{EtBr}$, when used at low concentration, also induces frame shift mutations and deletions ${ }^{44}$ and we demonstrated that it also triggers oxidative damage in the mtDNA. Our data show that mtDNA depletion induced by damage is caused by a specific exacerbated mtDNA turnover which is Atg5 dependent and thus requires autophagosome formation and lysosomal degradation. Interestingly, K320E localizes preferentially in specific mitochondrial regions. K320E localization does not overlap completely with the mitochondrial outer membrane protein TOM20 and is present in areas that are also not stained by Mitotracker Green, demonstrating that nucleoids containing Twinkle can be specifically localized in a unique mitochondrial subcompartment. Indeed, mtDNA has been shown to attach preferentially to cholesterol-rich membrane structures and Twinkle, as a nucleoid protein, has been found to be enriched in these areas as well ${ }^{45}$. The onion-like structures we observed in our cell model have been detected in the muscle of patients with mitochondrial myopathy caused by mtDNA mutations ${ }^{46}$ and very recently also in $\rho 0$-cells lacking $m t D N A{ }^{47}$. Here we show that mtDNA damage induces cristae remodelling in poles of the mitochondrial network and that these structures containing Twinkle are delivered to lysosomal compartments, which are therefore specifically involved in mtDNA removal.

396 Three proteins present in three mitochondrial compartments are responsible for mtDNA distribution and selective turnover: Twinkle, in the mitochondrial matrix; ATAD3, in the 
mitochondrial inner membrane and SAMM50, in the mitochondrial outer membrane. Twinkle arises as the link between nucleoids and the inner membrane through interaction with ATAD3, a protein controlling several aspects of mitochondrial membrane dynamics ${ }^{48}$. Interestingly, the human ortholog ATAD3B has been recently found to be a mitophagy receptor for mtDNA damage induced by oxidative stress ${ }^{49}$. In the other side, SAMM50, which resides in the mitochondrial outer membrane, interacts with the MICOS complex, and organizes membrane architecture ${ }^{50}$. Interestingly, both ATAD3 and SAMM50 have been described as regulators of Pink1-Parkin as well ${ }^{34,51}$, thus providing the link between nucleoid localization and specific degradation of mtDNA. Furthermore, we show that upon mtDNA damage, VPS35, an endosomal protein involved in mitochondrial quality control, increases contacts to mitochondrial subcompartments containing Twinkle. Interestingly, VPS35 deficient cells showed a persistent activation of mitophagy with mtDNA depletion. The fact that Samm50 depletion induces mitophagy, but excludes mtDNA degradation ${ }^{34}$, suggests that SAMM50 confers the required selectivity and VPS35 the specificity

411 to remove only fragments containing mtDNA.

412 Removal of mitochondrial fragments has been shown to take part through a specialized pathway 413 called Mitochondrial Derived Vesicles (MDVs) ${ }^{7}$. Currently, only a few proteins have been found 414 to determine cargo and vesicle fate. While MAPL generates vesicles which divert oxidized cargo 415 to peroxisomes ${ }^{32}$, Tollip, an endosomal organizer, synchronizes Parkin-dependent MDVs 416 directed to the lysosomal compartment ${ }^{8}$. Canonical MDVs are generated in the mitochondria in 417 an Atg5 and LC3 independent manner ${ }^{32}$, however, mtDNA damage by K320E expression 418 showed accumulation of LC3 autophagosomes and mtDNA depletion which is Atg5 dependent. 419 Additionally, electron microscopic pictures of our cells never showed vesicles resembling MDVs, 420 which are much smaller than the mitochondrial extrusions and autolysosomes containing Twinkle 421 we observed. Furthermore, we unequivocally showed that Twinkle particles are directed to the 422 lysosomal compartment and that VPS35 associates with mitochondria upon mtDNA damage. We 423 also showed that VPS35 recruitment is SAMM50 dependent and, upon mtDNA damage, such 424 recruitment is specific to Twinkle containing regions. Upon $\mathrm{EtBr}$ damage, we confirmed that 425 VPS35 precipitates with mitochondrial outer membrane proteins, such as SAMM50, TOMM40, 426 TIMMDC1 and VDAC1 and VDAC3 and it is in this environment when Twinkle, a mitochondrial 427 matrix protein, interacts with SAMM50.

428 mtDNA removal exemplifies a specialized mitophagy trail. Delivery of specific mitochondrial cargo 429 to quality control pathways through VPS35 was previously shown to be highly regulated. If the 430 selected cargo is diverted into peroxisomes, VPS35 associates with MDVs by interacting with 431 MAPL in a Drp1 independent manner ${ }^{10}$. However, if the MDVs are directed to lysosomes the 432 association is dependent on Drp1 ${ }^{52}$. On the other hand, SAMM50 modifies mitochondrial 433 structure by interacting with Drp $1^{53}$ and with the Pink1-Parkin machinery for selective mitophagy $434{ }^{34}$. Hence, SAMM50 works as a platform where mitochondrial fission, membrane architecture, 435 and mitophagy components orchestrate membrane protrusions and facilitate VPS35 recruitment. 436 Very recently, SAMM50 has been linked also to piecemeal mitophagy, independent of MDVs, 437 through direct interaction with the p62 adaptor ${ }^{54}$. Hence, we conclude that specific mtDNA 
degradation does not follow the MDV pathway, despite the fact that they share some components,

439 and that it is more likely that mitochondrial protrusions containing nucleoids are engulfed in 440 autophagosomes in a specialized mitophagy pathway.

441 VPS35, which has been extensively linked to neurological diseases such as Parkinson's and

442 Alzheimer disease, appears as a regulator of mtDNA quality control necessary to maintain

443 mitochondrial intactness. Modulation of VPS35 expression has been evaluated as a potential

444 approach against Parkinson's disease ${ }^{55}$ and, in Drosophila, Vps35 overexpression can rescue

445 an LRRK2-induced Parkinson's phenotype ${ }^{56}$. iPSC derived neurons from LRKK2-PD patients

446 showed accumulation of mtDNA damage ${ }^{57}$. Nonetheless, the ability of VPS35 to eliminate such

447 molecules in a human disease related model needs to be further explored as a potential 448 therapeutic strategy.

449 Enrichment of Spg7 upon VPS35 IP in EtBr treated cells also suggests the involvement of 450 mitochondrial proteases. Mitochondrial matrix proteases (mAAAs) represent a group of enzymes 451 related to mitochondrial quality control and mitochondrial membrane remodeling upon proteolytic 452 cleavage of Opa1 and Oma1. Spg7 has been found to copurify with Prohibitin participating in the 453 formation of the permeability transition pore ${ }^{58,59}$. Mutations in the SPG7 gene are the cause for 454 Hereditary Spastic Paraplegia Type 7 but also a Progressive External Optalmoplegia-like 455 syndrome with accumulation of mtDNA deletions ${ }^{60}$. Hence, it is tempting to propose that Spg7 456 works as a regulator of mtDNA turnover as well. However, further studies are needed to reveal 457 its specific role and regulation of the process.

458 Finally, our data demonstrate that specific removal of mtDNA is linked to lysosomal activity. 459 Lysosomal degradation of bulk autophagy is regulated by the serine-threonine protein kinase 460 mTORC1 which resides on the lysosomal surface. mtDNA replication defects activate mTORC1 461 and the integrated mitochondrial stress response in a cascade of effects with wide downstream 462 consequences ${ }^{61}$. It is well known that mTORC1 activation inhibits autophagy by influencing both 463 the formation of autophagosomes but also lysosomal acidification ${ }^{62}$. In order to prove the 464 selectivity of this process in vivo, we used a mouse model where mtDNA alterations rapidly 465 accumulate and indeed, activation of the mTORC1 pathway by rapamycin was able to eliminate 466 abnormal mtDNA molecules and thus reduces the accumulation of cells with mitochondrial 467 dysfunction. Rapamycin has been described as a potential treatment against mitochondrial 468 diseases ${ }^{18,63}$, however, since we observed a fiber-type shift, mTORC1 inhibition might activate 469 other signalling pathways with undesirable effects. Nonetheless, by using Rapamycin we 470 demonstrated that elimination of mutant mtDNA without affecting total mtDNA copy number is 471 possible.

472 In conclusion, we unveil a new complex mechanism with physiological relevance for mitochondrial 473 fitness. Twinkle mediates nucleoid binding to the mitochondrial inner membrane through ATAD3 474 interaction, which is responsible for nucleoid organization. SAMM50 provides the required 475 specificity to eliminate mtDNA while VPS35 supplies the selectivity. Interestingly, mutations in 476 TWNK, ATAD3A and VPS35 have been linked to several severe mitochondrial diseases having 477 in common mtDNA instability ${ }^{64-66}$, therefore representing a cluster of proteins involved in specific 
mtDNA turnover. Fine tuning the activity of such proteins could be used as a therapeutic strategy against mtDNA related diseases, either inherited, acquired or due to normal ageing.

\section{Methods}

482

\section{In vivo experimental approaches}

$484 \mathrm{~K} 320 \mathrm{E}$ transgenic mice were generated by crossing R26-K320EloxP/+ mice (point mutation 485 K320E; Rosa26-Stop-construct; downstream EGFP) with mice expressing Cre recombinase 486 under the control of the skeletal muscle-specific MLC1f- promoter or satellite cells Pax7-Cre ${ }^{\text {ERT }}$. 487 All mice used for experiments were housed in a standard animal facility maintained at $23^{\circ} \mathrm{C}$, 488 12:12h light-dark cycle, with free access to water and standard rodent chow. Autophagy flux was tested by intraperitoneal injection of $50 \mathrm{mg} / \mathrm{kg}$ chloroquine $4 \mathrm{~h}$ prior euthanasia. All procedures and experimentation with mice were performed according to protocols approved by the local authority (LANUV, Landesamt für Natur, Umwelt und Verbraucherschutz NRW, approval number: 2019A090). Activation of Pax7-Cre ${ }^{E R T}$ promotor was performed by injecting daily, for 5 days, intraperitoneal $2 \mathrm{mg}$ tamoxifen dissolved in mygliol. For muscle regeneration experiments, 2 days after the last tamoxifen injection, mice were anesthetized with $2 \%$ Xylazin, $10 \%$ Ketamine in $\mathrm{NaCl}$ $0.9 \%$ and $10 \mu \mathrm{M}$ Cardiotoxin (Naja Pallida, Latoxan) was injected inside the fascia. After 2 days of rest, $2 \mathrm{mg} / \mathrm{kg}$ rapamycin dissolved in mygliol was injected intraperitoneally daily for 5 days. The day after the last injection, mice were treated as explained below.

498

\section{Molecular biology and vector generation}

500 Twnk ORF was amplified from plasmids pJet2-Twinkle and pROSA-K320E ${ }^{67}$ and cloned in 501 pmCherry-N1. For retroviral vector generation, Twinkle and Twinkle-mCherry, ORF was amplified 502 and subcloned into pLenti-CMV Puro DEST (Addgene \#17452). All ORFs were subcloned into 503 pBabe-Puro vector, kindly provided by Dr. Bernhard Schermer, and verified by Sanger 504 sequencing. pLX304-TWINKLE-APEX2 vector was kindly provided by Dr. Alice Ting. To generate 505 Mus musculus Twnk vectors, Homo sapines TWNK was replaced by Twnk ORF and subcloned 506 into pBABE-Puro vector. Mitochondrial matrix APEX2 control vector (Addgene \#72480) was also 507 subcloned into pBABE-Puro. For generation of Tfam-GFP, total RNA from mouse liver was 508 isolated and converted into cDNA using TRIzol (Thermo Fisher) and RevertAid First Strand cDNA 509 Synthesis Kit (Thermo Fisher) using PolyT as feeder. Tfam cDNA was cloned into pEGFP-N1. 510 Atad3 KD and Samm50 KD clones were generated by transducing MEFs with the vector pMKO1511 GFP (Addgene \#10676) containing a shRNA (Table M1). Empty vector and a scramble containing 512 vector were used as a control. For CRISPR Cas9 KO generation two gRNA directed to exon 4 513 and exon 5 (Table M1) were cloned in pSpCas9 (BB)-2A-Puro V2.0 (Addgene \#62988).

\section{Generation and culture of cell lines}

516 C2C12 cell line was purchased from ATTC and grown in DMEM $4.5 \mathrm{~g} / \mathrm{L}$ Glucose + GlutaMax, $51720 \%$ FBS, 1x Pen/Strep. Immortalized mouse embryonic fibroblast Atg5WT and Atg5KO MEFs 
$518{ }^{68}$ and immortalized MEFs line were maintained as described ${ }^{69}$. Stable cell lines were generated 519 by transducing $\mathrm{C} 2 \mathrm{C} 12$ cells or MEFs with pBABE-Puro retroviruses. Briefly, $2.5 \times 10^{6} \mathrm{HEK} 293$ cells 520 were plated in $10 \mathrm{~cm}^{2}$ dish transfected with pCL-ECO $(5 \mu \mathrm{g})$ and pBABE-Puro $(10 \mu \mathrm{g})$ vectors 521 using PEI $(40 \mu \mathrm{l})$. After $48 \mathrm{~h}$ and $72 \mathrm{~h}$, medium containing viruses was harvested, filtered through $52245 \mu \mathrm{m}$, mixed with $8 \mu \mathrm{g} / \mathrm{ml}$ Polybrene and added to 250.000 cells previously plated. $48 \mathrm{~h}$ post 523 transduction, positive clones were selected by adding Puromycin $2.5 \mu \mathrm{g} / \mathrm{ml}$ to the medium in $524 \mathrm{C} 2 \mathrm{C} 12$ and $5 \mu \mathrm{g} / \mathrm{ml}$ to MEFs, which was maintained during all the experiments. shRNA clones 525 were generated by transducing MEFs with pMKO.1-GFP vectors. Prior to all experiments, 526 transduction rate was verified to be higher than 99\% of GFP expressing cells by Flow cytometry. 527 For generation of Vps35 CRISPR Cas9 KO clones, MEFs were transiently transfected with the 528 vector containing gRNA and selected with $3 \mu \mathrm{g} / \mathrm{ml}$ puromycin for 4 days. Single clones were 529 plated independently using cloning cylinders, analyzed by western blotting. Genomic DNA was 530 isolated from VPS35-negative cells and genomic DNA modification was verified by Sanger 531 sequencing. Exon 4 and Exon 5 were amplified (Table M1) and cloned using pJET1.2 cloning kit 532 (Thermo Fisher) before sequencing.

533 Transient transfection was achieved transfecting the corresponding plasmids using Lipofectamine 5343000 following manufacturer instructions. Plasmids use in this work were: LC3-GFP (Addgene 535 \#21073), Lamp1-GFP (Addgene \#34831), Fis1p-Cherry-GFP 70, LC3-Fis1p-Cherry (kindly 536 provided by Dr. Terje Johansen) and MAPL-GFP from CECAD Imaging Facility ${ }^{32}$.

537 For mtDNA depletion experiments, cells were maintained as described before for 7 days but 538 media was supplemented with $50 \mathrm{ng} / \mathrm{ml}$ Ethidium and $50 \mu \mathrm{g} / \mathrm{ml}$ Uridine. Lysosomal function was 539 blocked using $10 \mu \mathrm{M}$ Chloroquine for $24 \mathrm{~h}$.

540

\section{1 mtDNA amplification}

542 Total DNA was isolated using DNeasy Blood \& Tissue Kit (Qiagen) according to manufacturer's 543 instruction. $25 \mathrm{ng}$ of total DNA was used for analysis of threshold amplification differences 544 between mtDNA and nuclear DNA (delta $C(t)$ method with specific primers (Table M1). Long 545 range PCR was used to screen for the presence of mtDNA alterations. $14 \mathrm{~Kb}$ of mtDNA was 546 amplified using Rabbit Bioscience Long Range kit with oligos described in table M1.

547

\section{Western blot and co-immunoprecipitation}

549 Cells pellets were lysed with RIPA-buffer (150 mM NaCl, 1\% Triton-X1000, 0.1\% SDS, $50 \mathrm{mM}$ 550 Tris- $\mathrm{HCl} \mathrm{pH} \mathrm{8,0.5 \%} \mathrm{Na-deoxycholate)} \mathrm{containing} \mathrm{protease} \mathrm{inhibitor} \mathrm{(Roche)} \mathrm{and} \mathrm{protein}$ 551 concentration measured using the Bradford assay. Proteins were transferred after 552 electrophoresis to a PVDF-membrane previously activated with methanol. Membranes were 553 blocked (5\% milk in TBS-0.1\% Tween-20) and incubated overnight with primary antibodies. 554 Antibodies used in this work are: monoclonal $\alpha-\mathrm{V} 5$ (Abcam), polyclonal $\alpha-\mathrm{V} 5$ (Thermo Scientific), 555 polyclonal $\alpha$-TOM20 and monoclonal a-VPS35 (Santa Cruz), monoclonal and polyclonal $\alpha-$ 556 SAMM50 (Abnova and Abcam respectively), polyclonal a-ATAD3, polyclonal $\alpha$-LC3 and 557 polyclonal a-p62 (Proteintech), polyclonal GAPDH (Novus Biologicals). Secondary goat anti- 
mouse, goat anti-rabbit and goat anti-chicken HRP (Jackson Laboratory). Images were acquired using the ECL Advanced Chemiluminescence kit (GE Healthcare Life Sciences ${ }^{\circledR}$, UK) according to manufacturer's protocols and visualized using a LAS500 CCD camera.

561 For immunoprecipitation, cells expressing Twinkle-APEX2-V5 were pelleted and solubilized in IP 562 Buffer (500 mM HEPES KOH pH 7.2, 150 mM NaCl, 1 mM MgCl, $1 \%$ Triton-X1000 and Protease 563 inhibitor (Roche). $500 \mu \mathrm{g}$ of total protein extract were used to IP with either $2.5 \mu \mathrm{g}$ V5 rabbit 564 polyclonal antibody or VPS35 mouse monoclonal over night at $4^{\circ} \mathrm{C}$ and recovered after incubating

565 for $6 \mathrm{~h}$ at $4^{\circ} \mathrm{C}$ in a rotator with equilibrated Agarose Protein-G beads (Abcam). Immunoprecipitation followed by MS analysis was performed as described but using magnetic Protein $G$ beads (Thermo Scientific). Samples were washed 5 times with washing buffer (10 mM HEPES KOH pH $7.2,150 \mathrm{mM} \mathrm{NaCl}, 1 \mathrm{mM} \mathrm{MgCl} 2,0.2 \%$ Triton-X1000) and once with PBS. Prior to analysis by MS, samples were washed $3 x$ with ammonium-bicarbonate $(A B C)$ buffer, denatured with $50 \mu$ of urea buffer ( $6 \mathrm{M}$ urea, $2 \mathrm{M}$ thiourea) and followed by disulfide-bridge reduction using dithiothreitol (DTT) at a final concentration of $5 \mathrm{mM}$ for 1 hour at room temperature. To alkylate oxidized cysteines, 2-lodoacetamide (IAA) was added to the samples until a concentration of $40 \mathrm{mM}$ was reached and incubated for $30 \mathrm{~min}$ in the dark. Lys-C was added in a ratio of 1:100 (0.1 $\mu \mathrm{g}$ enzyme for $10 \mu \mathrm{g}$ protein) and incubated for 2-3 hours. Samples were finally diluted with ABC buffer to reach $2 M$ urea concentration. Protein digestion was performed overnight with trypsin 1:100. Samples were acidified with $1 \%$ formic acid and desalted using a modified version of the previously described Stop and Go extraction tip (StageTip) protocol ${ }^{71}$.

\section{Pulsed SILAC labeling in mice and in-solution digestion}

For pulsed SILAC labeling mice, 30-40 weeks old mice for K320E; Mlc1 line (C57BL/6J) were fed a ${ }^{13} \mathrm{C}_{6}$-lysine (Lys6)-containing mouse diet (Silantes) for 14 days to monitor newly synthesized proteins by comparing the incorporation of Lys- 6 with the naturally occurring Lys- $0{ }^{72}$. Mice were sacrificed at the end of day 14 and tissues dissected and snap-frozen in liquid nitrogen. Samples were grinded and proteins extracted and denatured by the addition of $4 \%$ SDS in PBS. To remove residual SDS proteins were precipitated overnight in $4 x$ ice-cold acetone (v:v). On the next day after centrifugation at $16,000 \mathrm{~g}$ for $10 \mathrm{~min}$ the protein pellets were dissolved in urea buffer $(6 \mathrm{M}$ urea / $2 \mathrm{M}$ thiourea). The following protein digestion was performed as described previously but instead of overnight tryptic digestion proteins were only digested with Lys-C (1:100 enzyme-toprotein ratio) for both pre-digestion ( $2 \mathrm{~h}$ at $\mathrm{RT}$ ) and overnight digestion after dilution of urea using $A B C$ buffer.

\section{Liquid chromatography - mass spectrometric analysis}

Both affinity-enriched and pulsed SILAC proteomics samples were analyzed in positive mode using data-dependent acquisition (DDA) either by an Easy-nLC $1000-Q$ Exactive Plus or an Easy-nLC 1200 - Orbitrap Eclipse tribrid system (all Thermo Fisher). On-line chromatography was directly coupled to the mass spectrometric systems using a nanoelectrospray ionization source. Peptides were separated by reversed-phase chromatography with a binary buffer system 
of buffer $\mathrm{A}$ ( $0.1 \%$ formic acid in water) and buffer $\mathrm{B}(0.1 \%$ formic acid in $80 \%$ acetonitrile) using a $60 \mathrm{~min}$ chromatographic gradient for IP samples and $120 \mathrm{~min}$ for pulsed SILAC samples.

600 Separation was performed on a $50 \mathrm{~cm}$ long in-house packed analytical column filled with $1.9 \mu \mathrm{M}$

601 C18-AQ Reprosil Pur beads (Dr. Maisch). Using the 60 min chromatographic gradient peptide separation based on their hydrophobicity was performed by linearly increasing the amount of 603 buffer B from initial $13 \%$ to $48 \%$ over $35 \mathrm{~min}$ followed by an increase of B to $95 \%$ for $10 \mathrm{~min}$. The column was washed for $5 \mathrm{~min}$ and initial column conditions were achieved by equilibrating the column for $10 \mathrm{~min}$ at $7 \%$ B. Full MS spectra (300 to $1750 \mathrm{~m} / \mathrm{z}$ ) were acquired with a resolution of 70,000 , a maximum injection time of $20 \mathrm{~ms}$ and an AGC target of 3e6. The top 10 most abundant peptide ions were isolated $(1.8 \mathrm{~m} / \mathrm{z}$ isolation windows) for subsequent HCD fragmentation (NCE

$608=28$ ) and MS/MS recording at a resolution of 35,000, a maximum injection time of 120 ms and an AGC target of $5 \mathrm{e} 5$. Peptide ions selected for fragmentation were dynamically excluded for 20 seconds.

611 Using the 120 min chromatographic gradient peptides were separated by linearly increasing B 612 from initial $4 \%$ to $25 \%$ over 96 min followed by an increase of B to $55 \%$ over $14 \mathrm{~min}$. After a steep 613 increase of B to $95 \%$ over 2 min the analytical column was washed for 8 min at $95 \%$ B. Full MS 614 spectra ( 375 to $1500 \mathrm{~m} / \mathrm{z}$ ) were acquired with a resolution of 60,000 , a dynamic injection time and 615 an automated AGC target. The top 20 most abundant peptide ions (charge state $2-7$ ) were 616 isolated (1.2 m/z isolation windows) for subsequent HCD fragmentation (NCE = 30) and MS/MS 617 recording at a resolution of 15,000, a maximum injection time of $22 \mathrm{~ms}$ and an automated AGC target. Peptide ions selected for fragmentation were dynamically excluded for 60 seconds.

\section{Data processing and analysis}

621 The mass spectrometry proteomics data have been deposited to the ProteomeXchange 622 Consortium (http://proteomecentral.proteomexchange.org) via the PRIDE partner repository ${ }^{73}$ with the dataset identifier PXD023939. All recorded RAW files were processed with the MaxQuant software suite (1.5.3.8 for IP data, 1.6.14 for pSILAC) ${ }^{74}$. For peptide identification and scoring MS/MS spectra were matched against the mouse Uniprot database (downloaded 08/15/2019) using the Andromeda search algorithm ${ }^{75}$. For the affinity-enriched samples multiplicity was set to one and trypsin/P was selected as digestive enzyme. Carbamidomethylation was set as a fixed modification and methionine oxidation or $\mathrm{N}$-terminal acetylation was selected as variable modification. Peptides were identified with a minimum amino acid length of seven and a falsediscovery rate (FDR) cut-off of $1 \%$ on the peptide level. Proteins were identified with FDR $<1 \%$ using unique and razor peptides for quantification. Label-free quantification was performed using the standard settings of the maxLFQ algorithm. Match between runs was activated. pSILAC data were analyzed with the same settings with some modifications. Multiplicity was set to two with Lys6 as heavy isotope label. Lys-C/P was selected as digestive peptide and LFQ quantification was deactivated.

636 Statistical analysis and visualization were done with the Perseus (1.6.5) and InstantClue software 637 suits 76,77 . LFQ intensities of the IP samples were $\log _{2}$-transformed and filtered for proteins 
identified in all replicates of at least one condition. Missing values were imputed by random drawing of values from 1.8 standard deviations (SD) downshifted, 0.3 SD broad normal distribution to simulate the lower detection limits of the mass spectrometer. To evaluate principal components responsible for the variances between samples we performed a principal component analysis. Further, we performed a two-sided Student's $t$-test to identify significantly regulated proteins. We proceeded similarly for the pulsed SILAC data but used the heavy-to-light $(H / L)$ ratios for statistical testing. We used the same filtering-criteria but did not imputed missing values. We performed a 1D annotation enrichment to identify enriched categorical terms in the different conditions $^{78}$.

647

\section{Histology, Immunofluorescence and Microscopy}

649 For tissue histology, mice were sacrificed by cervical dislocation, muscles dissected, mounted in cork with OCT (Tissue-Tek), snap frozen in isopentane and stored at $-80^{\circ} \mathrm{C}$ till needed. $10 \mu \mathrm{m}$ thick sections covering the injured area were produced using a cryostat maintained at $-20^{\circ} \mathrm{C}$ (Leica CM 3050s, Techno-med). To assess the integrity of mitochondrial function the sections were sequentially stained for $\mathrm{COX}$ and SDH activities. Frozen sections were incubated 20 mins at $37^{\circ} \mathrm{C}$ in COX solution $(20 \mathrm{mg} / \mathrm{ml}$ catalase, $74 \mathrm{mg} / \mathrm{ml}$ sucrose, $2 \mathrm{mg} / \mathrm{ml}$ cytochrome $\mathrm{c}$, and $1 \mathrm{mg} / \mathrm{ml} \mathrm{DAB}$ in $50 \mathrm{mM} \mathrm{Na}_{2} \mathrm{HPO}_{4} \mathrm{pH} 7.4$ ). After 3 PBS washes, sections were then incubated for $30 \mathrm{~min}$ at $37^{\circ} \mathrm{C}$ in $\mathrm{SDH}$ staining solution $(2 \mathrm{mg} / \mathrm{ml} \mathrm{NBT}, 0.2 \mathrm{M}$ Sodium succinate, $50 \mathrm{mM} \mathrm{MgCl}$, $50 \mathrm{mM}$ Tris- $\mathrm{HCl}, \mathrm{pH} 7.4$ ), washed 3 times with miliQ water, and mounted in Glycerol gelatin medium (Sigma).

659 For immunofluorescence of autophagy markers LC3 and p62 and mitochondrial TOM20, mice 660 were perfused with PFA 4\% in PBS prior to muscle collection. Samples were equilibrated in 15\% 661 sucrose for $6 \mathrm{~h}$ and $30 \%$ sucrose overnight before frozen in OCT medium. For LC3 IF, samples 662 were preincubated with $0.1 \%$ SDS for $5 \mathrm{~min}$. Antibody specificity was determined in muscle sections from LC3-GFP transgenic mice (kindly provided by Dr. Evangelos Kondilis).

664 Cryosections were blocked for $1 \mathrm{~h}$ with $1 \%$ Western blocking reagent (Roche) containing $0.1 \%$ 665 Triton in PBST, antibodies incubated overnight at $4^{\circ} \mathrm{C}$ and secondary antibodies at room 666 temperature for $1 \mathrm{~h}$ in blocking buffer. Samples were mounted in Fluoromount $\mathrm{G}$ containing DAPI. 667 Fiber type staining was performed as described previously ${ }^{26}$. Images were obtained with Leica 668 SP8 with $63 x / 1.40$ oil PL Apo objective.

669 For in vitro analysis, cells were fixed in 4\% PFA/PBS, permeabilized with PBS-0.2\% Triton-X1000 670 for 30 min and blocked for 1 hour at RT in blocking buffer ( $5 \%$ fat free milk powder, $10 \% \mathrm{FBS}, 1 \%$ 671 BSA, 0.1\% Triton-X100 in PBS). Primary antibodies were incubated in blocking buffer over night 672 at $4^{\circ} \mathrm{C}$ and secondary antibodies for 1 hour at RT. mtDNA replication rate was determined by 673 pulse BrdU labelling. Briefly, cells were incubated with $20 \mu \mathrm{M} \mathrm{BrdU}$ (Sigma) for $6 \mathrm{~h}$, fixed with $4 \%$ 674 PFA/PBS for 30 minutes and directly permeabilize with $05 \%$ Triton X-100 on ice for 5 min. To 675 allow access to mtDNA, cells were incubated with $\mathrm{HCl} 2 \mathrm{~N}$ for another 30 min prior to 676 immunofluorescence. Coverslips were mounted using DAPI-Fluoromount G. Images were 677 acquired using a spinning-disk confocal microscope (Ultra View VoX; PerkinElmer) with a Plan- 
684 Antibodies used for immunofluorescence were: rabbit polyclonal $\alpha-\mathrm{V} 5$ (Thermo); rabbit polyclonal $685 \alpha-T O M 20$, mouse monoclonal $\alpha-V s p 35$ and mouse monoclonal $\alpha-8-O H d G$ (Santa Cruz); goat 686 polyclonal $\alpha-V s p 35$ and mouse monoclonal $\alpha$-dsDNA (Abcam); rabbit polyclonal $\alpha$-ATAD3, rabbit 687 polyclonal $\alpha-L C 3$, rabbit polyclonal $\alpha-p 62$, rabbit polyclonal LRPPRC (Proteintech); and 688 monoclonal $\alpha$-BrdU (BD Bioscience). Fluorescence secondary antibodies goat $\alpha$-mouse, $\alpha$-rabbit 689 Alexa Fluor-488, 555 and 647 and rabbit a-goat-647 were used accordingly to the primary 690 antibodies. Additionally, a-mouse IgM Alexa Fluor 488, a-mouse IgG Alexa Fluor 555 and $\alpha$ 691 mouse IgG2b Alexa Fluor 647 were used for fiber type triple staining.

692

\section{Electron microscopy}

694 For electron microscope cells were grown on small discs of aclar foil and fixed for $1 \mathrm{~h}$ in $2 \%$ 695 Glutaraldehyde with $2 \%$ Sucrose in HEPES buffer $\mathrm{pH} 7,4$. After washing two times with $0.1 \mathrm{M}$ 696 Cacodylate buffer, free aldehyde groups were quenched with $0.1 \mathrm{M}$ Glycin in $0.1 \mathrm{M}$ Cacodylate 697 buffer for two times $20 \mathrm{~min}$. After a short wash with $0.1 \mathrm{M}$ Cacodylate buffer, cells were incubated 698 in $0.5 \mathrm{mg} / \mathrm{ml}$ Diaminobenzidine in $0.1 \mathrm{M}$ Cacodylate buffer and, after $10 \mathrm{~min}$, a final concentration 699 of $0.03 \% \mathrm{H}_{2} \mathrm{O}_{2}$ added and incubated for $30 \mathrm{~min}$. Finally, cells were washed three times with $0.1 \mathrm{M}$ 700 Cacodylate buffer and incubated with 1\% Osmiumtetroxid and 1.5\% Potassium hexacyanoferrat 701 for $30 \mathrm{~min}$ at $4^{\circ} \mathrm{C}$. After $3 \times 5 \mathrm{~min}$ wash with $\mathrm{ddH} 2 \mathrm{O}$, samples were dehydrated using ascending 702 ethanol series $(50 \%, 70 \%, 90 \%, 100 \%)$ for 5 min each and infiltrated with a mixture of $50 \%$ 703 Epon/ethanol overnight at $4^{\circ} \mathrm{C}$ and with pure Epon for two times $2 \mathrm{~h}$. Samples were embedded 704 into TAAB capsules and cured for $48 \mathrm{~h}$ at $60^{\circ} \mathrm{C}$.

705 For electron tomography Ultrathin sections of $200 \mathrm{~nm}$ were cut using an ultramicrotome (Leica, $706 \mathrm{UC7}$ ) and incubated with $10 \mathrm{~nm}$ protein A gold (CMC, Utrecht) diluted 1:25 in ddH20. Sections 707 were stained with $2 \%$ Uranyl acetate for $20 \mathrm{~min}$ and Reynolds lead citrate solution for $3 \mathrm{~min}$. 708 Images and Tilt series for $1 \mathrm{~nm}$ thickness were acquired from $-65^{\circ}$ to $65^{\circ}$ with $1^{\circ}$ increment on a 709 JEM-2100 Plus Transmission Electron Microscope (JEOL) operating at 200kV equipped with a 710 OneView 4K 32 bit (Gatan) using SerialEM (Mastronarde, 2005). Reconstruction was done using 711 Imod (Kremer et al.,1996).

712

\section{Image analysis}

714 All image analysis was performed in FIJI (NIH, Bethesda). LC3 and p62 puncta quantification 715 were performed with the "counting cells" internal plugin for particles bigger than 2 pixels to exclude 716 background. mtDNA foci quantification, VPS35-Twinkle analysis, 8-OHdG and BrdU analysis 717 were performed with a self-created macro. Briefly, threshold was set for the different channels. 
Nuclear signal was selected and removed for the analysis. The signal corresponding to the

719 mitochondrial network was selected and only the particles from the other channel bigger than 1

720 pixel inside the mitochondrial network were considered to the analysis. For VPS35, the minimum

721 size was determined to be 3 pixels. After thresholding, all VPS35 particles were summarized and

722 the ones in contact with Twinkle or LRPPRC were used to get a percentage. Manders' coefficient

723 was obtained using JaCOP plugin. (Bolte \& Cordelieres, 2006).

724

725 Table M1. Oligonucleotides used in this study.

\begin{tabular}{|l|l|}
\hline shRNA name & Sequence 5'-3' $^{\prime}$ \\
\hline shAtad3_1 & $\begin{array}{l}\text { AGCCTGTATAGGAACGTTCTTCTCGAGGAACGTTCCTATACAGGCTTT } \\
\text { TTTTTG }\end{array}$ \\
\hline shAtad3_2 & $\begin{array}{l}\text { GCCTGTATAGGAACGTTCTTTCTCGAGAGAACGTTCCTATACAGGCTT } \\
\text { TTTTTG }\end{array}$ \\
\hline shAtad3_3 & $\begin{array}{l}\text { GCAGTTTGATTGGGCTATCTTCTCGAGGATAGCCCAATCAAACTGCTT } \\
\text { TTTTTG }\end{array}$ \\
\hline shScramble & $\begin{array}{l}\text { CCTAAGGTTAAGTCGCCCTCGCTCGAGCGAGGGCGACTTAACCTTAG } \\
\text { GTTTTTG }\end{array}$ \\
\hline shSamm50 & $\begin{array}{l}\text { GAGGAGATGTGAGCTTCATTTCAAGAGAATGAAGCTCACATCTCCTCT } \\
\text { TTTTG }\end{array}$ \\
\hline mVps35-ex4-104fw-s & CACCGTATGAACTTGTACAGTACGC \\
\hline mVps35-ex4-104fw-as & AAACGCGTACTGTACAAGTTCATAC \\
\hline mVps35-ex5-91fw-s & CACCGATTTGGTAGAAATGTGCCG \\
\hline mVps35-ex5-91fw-as & AAACCGGCACATTTCTACCAAATC \\
\hline mtDNA qPCR Forward & CCTATCACCCTTGCCATCAT \\
\hline mtDNA qPCR Reverse & GAGGCTGTTGCTTGTGTGAC \\
\hline nucDNA qPCR Forward & ATGGAAAGCCTGCCATCATG \\
\hline nucDNA qPCR Reverse & TCCTTGTTGTTCAGCATCAC \\
\hline mitoLongRange Forward & GTTCAACGATTAAAGTCCTACGT \\
\hline mitoLongRange Reverse & GTTGTTTGATCCTGTTTCGTG \\
\hline Del983-4977 Forward & TCGTAACAAGGTAAGCATACTG \\
\hline Del983-4977 Reverse & CTCGCGGACTAGTATATCCT \\
\hline Vps35_Ex4 Forward & CTGAGCCAGGAGATCATGAATTC \\
\hline Vps35_Ex4 Reverse & GCTTCCACTACTGAGCTAGATCAC \\
\hline Vps35_Ex5 Forward & GTCTAGACACAACTTACTGACACC \\
\hline Vps35_Ex5 Reverse & GTAGTGTGTTTGAATACAGTCAAG \\
\hline
\end{tabular}

726

\section{Acknowledgements}

728 We are thankful to CECAD Imaging and proteomics facilities for excellent technical support. We 729 thank Nadine Niehoff and Katrin Lanz for technical assistance. We are grateful to Thomas Paß 730 for critical review and discussion of the results. This work was supported by grants from the 731 Deutsche Forschungs-gemeinschaft (PL 895/1-1) and Köln Fortune (341/2019) to DPM and RJW. 732 
734 Funding Acquisition, DPM and RJW; Conceptualization, DPM and RJW; Investigation and Formal 735 Analysis, DPM, AS, SK, KM, JH; Resources JN; Analysis of MS Data, SK; Visualization, DPM; 736 Writing, DPM and RJW; Writing-Review \& Edit; DPM, RJW, SK, MK; Supervision, DPM and MK.

737

738

\section{Conflict of interest}

739

The authors declare that they have no conflict of interest.

740

741

\section{References}

7421 Samuels, D. C. et al. Recurrent tissue-specific mtDNA mutations are common in humans. PLoS Genet 9, e1003929, doi:10.1371/journal.pgen.1003929 (2013).

745

2 Lopez-Otin, C., Blasco, M. A., Partridge, L., Serrano, M. \& Kroemer, G. The 746 hallmarks of aging. Cell 153, 1194-1217, doi:10.1016/j.cell.2013.05.039 (2013).

747

3 Stewart, J. B. \& Chinnery, P. F. Extreme heterogeneity of human mitochondrial DNA from organelles to populations. Nat Rev Genet, doi:10.1038/s41576-02000284-x (2020).

749

750

751

752

753

754

755

756

757

758

4 Pickles, S., Vigie, P. \& Youle, R. J. Mitophagy and Quality Control Mechanisms in doi:10.1016/j.cub.2018.01.004 (2018).

5 Chen, H. C. et al. Mitochondrial Fusion Is Required for mtDNA Stability in Skeletal Muscle and Tolerance of mtDNA Mutations. Cell 141, 280-289, doi:10.1016/j.cell.2010.02.026 (2010).

6 Silva Ramos, E. et al. Mitochondrial fusion is required for regulation of mitochondrial DNA replication. PLoS Genet 15, e1008085, doi:10.1371/journal.pgen.1008085 (2019).

759

760

761

762

763

764

765

766

767

768

769

770

771

772

7 Sugiura, A., McLelland, G. L., Fon, E. A. \& McBride, H. M. A new pathway for mitochondrial quality control: mitochondrial-derived vesicles. EMBO J 33, 21422156, doi:10.15252/embj.201488104 (2014).

8 Ryan, T. A. et al. Tollip coordinates Parkin-dependent trafficking of mitochondrial-derived vesicles. Embo Journal, doi:ARTN e10253910.15252/embj.2019102539 (2020).

9 McLelland, G. L., Soubannier, V., Chen, C. X., McBride, H. M. \& Fon, E. A. Parkin and PINK1 function in a vesicular trafficking pathway regulating mitochondrial quality control. EMBO J 33, 282-295, doi:10.1002/embj.201385902 (2014).

10 Braschi, E. et al. Vps35 Mediates Vesicle Transport between the Mitochondria and Peroxisomes. Current Biology 20, 1310-1315, doi:10.1016/j.cub.2010.05.066 (2010).

11 Williams, E. T. et al. Parkin mediates the ubiquitination of VPS35 and modulates retromer-dependent endosomal sorting. Human Molecular Genetics 27, 31893205, doi:10.1093/hmg/ddy224 (2018).

12 Soubannier, V. et al. A vesicular transport pathway shuttles cargo from mitochondria to lysosomes. Curr Biol 22, 135-141, doi:10.1016/j.cub.2011.11.057 (2012).

13 Hanss, Z. et al. Mitochondrial and Clearance Impairment in p.D620N VPS35 Patient-Derived Neurons. Mov Disord, doi:10.1002/mds.28365 (2020). 
14 Neuhaus, J. F. et al. Catecholamine metabolism drives generation of mitochondrial DNA deletions in dopaminergic neurons. Brain 137, 354-365, doi:10.1093/brain/awt291 (2014).

15 Dolle, C. et al. Defective mitochondrial DNA homeostasis in the substantia nigra in Parkinson disease. Nat Commun 7, 13548, doi:10.1038/ncomms13548 (2016).

16 Peter, B. \& Falkenberg, M. TWINKLE and Other Human Mitochondrial DNA Helicases: Structure, Function and Disease. Genes (Basel) 11, doi:10.3390/genes11040408 (2020).

17 Bove, J., Martinez-Vicente, M. \& Vila, M. Fig.hting neurodegeneration with rapamycin: mechanistic insights. Nature Reviews Neuroscience 12, 437-452, doi:10.1038/nrn3068 (2011).

18 Civiletto, G. et al. Rapamycin rescues mitochondrial myopathy via coordinated activation of autophagy and lysosomal biogenesis. EMBO Mol Med 10, doi:10.15252/emmm.201708799 (2018).

791

792

19 Barriocanal-Casado, E. et al. Rapamycin administration is not a valid therapeutic

793

794

795

796

797

798

799

800

801

802

803

804

805

806

807

808

809

810

811

812

813

814

815

816

817

818

819

820 strategy for every case of mitochondrial disease. Ebiomedicine 42, 511-523, doi:10.1016/j.ebiom.2019.03.025 (2019).

20 Tyynismaa, $\mathrm{H}$. et al. Mutant mitochondrial helicase Twinkle causes multiple mtDNA deletions and a late-onset mitochondrial disease in mice. Proc Natl Acad Sci U S A 102, 17687-17692, doi:10.1073/pnas.0505551102 (2005).

21 Baris, O. R. et al. The mitochondrial electron transport chain is dispensable for proliferation and differentiation of epidermal progenitor cells. Stem Cells 29, 1459-1468, doi:10.1002/stem.695 (2011).

22 Oexner, R. R. et al. Extraocular Muscle Reveals Selective Vulnerability of Type IIB Fibers to Respiratory Chain Defects Induced by Mitochondrial DNA Alterations. Invest Ophthalmol Vis Sci 61, 14, doi:10.1167/iovs.61.12.14 (2020).

23 Weiland, D. et al. Imbalance of Mitochondrial Respiratory Chain Complexes in the Epidermis Induces Severe Skin Inflammation. J Invest Dermatol 138, 132-140, doi:10.1016/j.jid.2017.08.019 (2018).

24 Holzer, T. et al. Respiratory chain inactivation links cartilage-mediated growth retardation to mitochondrial diseases. J Cell Biol 218, 1853-1870, doi:10.1083/jcb.201809056 (2019).

25 Basu, S. et al. Accurate mapping of mitochondrial DNA deletions and duplications using deep sequencing. PLoS Genet 16, e1009242, doi:10.1371/journal.pgen.1009242 (2020).

26 Kallabis, S. et al. High-throughput proteomics fiber typing (ProFiT) for comprehensive characterization of single skeletal muscle fibers. Skelet Muscle 10, 7, doi:10.1186/s13395-020-00226-5 (2020).

27 Goffart, S. et al. Twinkle mutations associated with autosomal dominant progressive external ophthalmoplegia lead to impaired helicase function and in vivo mtDNA replication stalling. Hum Mol Genet 18, 328-340, doi:10.1093/hmg/ddn359 (2009).

28 Ock, C. Y. et al. 8-Hydroxydeoxyguanosine: not mere biomarker for oxidative stress, but remedy for oxidative stress-implicated gastrointestinal diseases. World J Gastroenterol 18, 302-308, doi:10.3748/wjg.v18.i4.302 (2012). 
29 Mai, S., Muster, B., Bereiter-Hahn, J. \& Jendrach, M. Autophagy proteins LC3B, ATG5 and ATG12 participate in quality control after mitochondrial damage and influence lifespan. Autophagy 8, 47-62, doi:10.4161/auto.8.1.18174 (2012).

30 Kleele, T. et al. Distinct fission signatures predict mitochondrial degradation or biogenesis. Nature 593, 435-439, doi:10.1038/s41586-021-03510-6 (2021).

$31 \mathrm{He}, \mathrm{J}$. Y. et al. The AAA(+) protein ATAD3 has displacement loop binding properties and is involved in mitochondrial nucleoid organization. Journal of Cell Biology 176, 141-146, doi:10.1083/jcb.200609158 (2007).

32 Neuspiel, M. et al. Cargo-selected transport from the mitochondria to peroxisomes is mediated by vesicular carriers. Current Biology 18, 102-108, doi:10.1016/j.cub.2007.12.038 (2008).

33 Ott, C. et al. Sam50 Functions in Mitochondrial Intermembrane Space Bridging and Biogenesis of Respiratory Complexes. Molecular and Cellular Biology 32, 1173-1188, doi:10.1128/Mcb.06388-11 (2012).

34 Jian, F. et al. Sam50 Regulates PINK1-Parkin-Mediated Mitophagy by Controlling PINK1 Stability and Mitochondrial Morphology. Cell Rep 23, 2989-3005, doi:10.1016/j.celrep.2018.05.015 (2018).

35 Sage-Schwaede, A. et al. Exploring mTOR inhibition as treatment for mitochondrial disease. Annals of Clinical and Translational Neurology 6, 18771881, doi:10.1002/acn3.50846 (2019).

36 Dai, Y. et al. Rapamycin drives selection against a pathogenic heteroplasmic mitochondrial DNA mutation. Human Molecular Genetics 23, 637-647, doi:10.1093/hmg/ddt450 (2014).

37 Kandul, N. P., Zhang, T., Hay, B. A. \& Guo, M. Selective removal of deletionbearing mitochondrial DNA in heteroplasmic Drosophila. Nat Commun 7, 13100, doi:10.1038/ncomms13100 (2016).

38 Szczepanowska, K. \& Trifunovic, A. in The Human Mitochondrial Genome (eds Giuseppe Gasparre \& Anna Maria Porcelli) 221-242 (Academic Press, 2020).

39 Alsina, D. et al. FBXL4 deficiency increases mitochondrial removal by autophagy. EMBO Mol Med 12, e11659, doi:10.15252/emmm.201911659 (2020).

40 Larsson, N. G. Somatic mitochondrial DNA mutations in mammalian aging. Annu Rev Biochem 79, 683-706, doi:10.1146/annurev-biochem-060408-093701 (2010).

41 Mishra, P., Varuzhanyan, G., Pham, A. H. \& Chan, D. C. Mitochondrial Dynamics Is a Distinguishing Feature of Skeletal Muscle Fiber Types and Regulates Organellar Compartmentalization. Cell Metabolism 22, 1033-1044, doi:10.1016/j.cmet.2015.09.027 (2015).

42 Crupi, A. N. et al. Oxidative muscles have better mitochondrial homeostasis than glycolytic muscles throughout life and maintain mitocnondrial function during aging. Aging-Us 10, 3327-3352, doi:10.18632/aging.101643 (2018).

43 Yang, X., Zhang, R., Nakahira, K. \& Gu, Z. Mitochondrial DNA Mutation, Diseases, and Nutrient-Regulated Mitophagy. Annu Rev Nutr 39, 201-226, doi:10.1146/annurev-nutr-082018-124643 (2019).

44 Yielding, L. W., Brown, B. R., Graves, D. E. \& Yielding, K. L. Ethidium-Bromide Enhancement of Frameshift Mutagenesis Caused by Photoactivatable Ethidium Analogs. Mutation Research 63, 225-232, doi:Doi 10.1016/0027-5107(79)900551 (1979). 
45 Gerhold, J. M. et al. Human Mitochondrial DNA-Protein Complexes Attach to a Cholesterol-Rich Membrane Structure (vol 5, 15292, 2015). Scientific Reports 5, doi:ARTN 17119

874

875

876

877

878

879

880

881

882

883

884

885

886

887

888

889

890

891

892

893

894

895

896

897

898

899

900

901

902

903

904

905

906

907

908

909

910

911

912

913

914

915

916

\subsection{8/srep17119 (2015).}

46 Vincent, A. E. et al. The Spectrum of Mitochondrial Ultrastructural Defects in Mitochondrial Myopathy. Scientific Reports 6, doi:ARTN 30610

10.1038/srep30610 (2016).

$47 \mathrm{Hu}, \mathrm{C}$. et al. OPA1 and MICOS Regulate mitochondrial crista dynamics and formation. Cell Death Dis 11, 940, doi:10.1038/s41419-020-03152-y (2020).

48 Peralta, S. et al. ATAD3 controls mitochondrial cristae structure in mouse muscle, influencing mtDNA replication and cholesterol levels. J Cell Sci 131, doi:10.1242/jcs.217075 (2018).

49 Shu, L. et al. ATAD3B is a mitophagy receptor mediating clearance of oxidative stress-induced damaged mitochondrial DNA. EMBO J 40, e106283, doi:10.15252/embj.2020106283 (2021).

50 Tang, J. H. et al. Sam50-Mic19-Mic60 axis determines mitochondrial cristae architecture by mediating mitochondrial outer and inner membrane contact. Cell Death and Differentiation 27, 146-160, doi:10.1038/s41418-019-0345-2 (2020).

51 Jin, G. et al. Atad3a suppresses Pink1-dependent mitophagy to maintain homeostasis of hematopoietic progenitor cells. Nat Immunol 19, 29-40, doi:10.1038/s41590-017-0002-1 (2018).

52 Wang, W. et al. Parkinson's disease-associated mutant VPS35 causes mitochondrial dysfunction by recycling DLP1 complexes. Nat Med 22, 54-63, doi:10.1038/nm.3983 (2016).

53 Liu, S. et al. SAMM50 Affects Mitochondrial Morphology through the Association of Drp1 in Mammalian Cells. Febs Letters 590, 1313-1323, doi:10.1002/18733468.12170 (2016).

54 Abudu, Y. P. et al. SAMM50 acts with p62 in piecemeal basal- and OXPHOSinduced mitophagy of SAM and MICOS components. I Cell Biol 220, doi:10.1083/jcb.202009092 (2021).

55 Eleuteri, S. \& Albanese, A. VPS35-Based Approach: A Potential Innovative Treatment in Parkinson's Disease. Front Neurol 10, 1272, doi:10.3389/fneur.2019.01272 (2019).

56 Linhart, R. et al. Vacuolar protein sorting 35 (Vps35) rescues locomotor deficits and shortened lifespan in Drosophila expressing a Parkinson's disease mutant of Leucine-rich repeat kinase 2 (LRRK2). Molecular Neurodegeneration 9, doi:Artn 2310.1186/1750-1326-9-23 (2014).

57 Sanders, L. H. et al. LRRK2 mutations cause mitochondrial DNA damage in iPSCderived neural cells from Parkinson's disease patients: Reversal by gene correction. Neurobiology of Disease 62, 381-386, doi:10.1016/j.nbd.2013.10.013 (2014).

58 Shanmughapriya, S. et al. SPG7 is an Essential and Conserved Component of the Mitochondrial Permeability transition Pore. Biophysical Journal 110, 309a-310a, doi:DOI 10.1016/j.bpj.2015.11.1663 (2016).

59 Sambri, I. et al. Impaired flickering of the permeability transition pore causes SPG7 spastic paraplegia. Ebiomedicine 61, doi:ARTN 10305010.1016/j.ebiom.2020.103050 (2020). 
91760 Pfeffer, G. et al. Mutations in the SPG7 gene cause chronic progressive external ophthalmoplegia through disordered mitochondrial DNA maintenance. Brain 137, 1323-1336, doi:10.1093/brain/awu060 (2014).

61 Khan, N. A. et al. mTORC1 Regulates Mitochondrial Integrated Stress Response and Mitochondrial Myopathy Progression. Cell Metabolism 26, 419-+, doi:10.1016/j.cmet.2017.07.007 (2017).

62 Dossou, A. S. \& Basu, A. The Emerging Roles of mTORC1 in Macromanaging Autophagy. Cancers 11, doi:ARTN 142210.3390/cancers11101422 (2019).

63 Ding, Y. et al. Rapamycin Ameliorates Cognitive Impairments and Alzheimer's Disease-Like Pathology with Restoring Mitochondrial Abnormality in the Hippocampus of Streptozotocin-Induced Diabetic Mice. Neurochem Res, doi:10.1007/s11064-020-03160-6 (2020).

Desai, R. et al. ATAD3 gene cluster deletions cause cerebellar dysfunction associated with altered mitochondrial DNA and cholesterol metabolism. Brain 140, 1595-1610, doi:10.1093/brain/awx094 (2017).

65 Deutschlander, A., Ross, O. A. \& Wszolek, Z. K. in GeneReviews((R)) (eds M. P. Adam et al.) (1993).

66 Remtulla, S., Emilie Nguyen, C. T., Prasad, C. \& Campbell, C. Twinkle-Associated Mitochondrial DNA Depletion. Pediatr Neurol 90, 61-65, doi:10.1016/j.pediatrneurol.2018.08.007 (2019).

67 Baris, O. R. et al. Mosaic Deficiency in Mitochondrial Oxidative Metabolism Promotes Cardiac Arrhythmia during Aging. Cell Metab 21, 667-677, doi:10.1016/j.cmet.2015.04.005 (2015).

68 Nuchel, J. et al. TGFB1 is secreted through an unconventional pathway dependent on the autophagic machinery and cytoskeletal regulators. Autophagy 14, 465-486, doi:10.1080/15548627.2017.1422850 (2018).

69 Gao, J. et al. CLUH regulates mitochondrial biogenesis by binding mRNAs of nuclear-encoded mitochondrial proteins. J Cell Biol 207, 213-223, doi:10.1083/jcb.201403129 (2014).

70 Allen, G. F. G., Toth, R., James, J. \& Ganley, I. G. Loss of iron triggers PINK1/Parkinindependent mitophagy. Embo Reports 14, 1127-1135, doi:10.1038/embor.2013.168 (2013).

71 Rappsilber, J., Ishihama, Y. \& Mann, M. Stop and go extraction tips for matrixassisted laser desorption/ionization, nanoelectrospray, and LC/MS sample pretreatment in proteomics. Anal Chem 75, 663-670, doi:10.1021/ac026117i (2003).

72 Kruger, M. et al. SILAC mouse for quantitative proteomics uncovers kindlin-3 as an essential factor for red blood cell function. Cell 134, 353-364, doi:10.1016/j.cell.2008.05.033 (2008).

73 Perez-Riverol, Y. et al. The PRIDE database and related tools and resources in 2019: improving support for quantification data. Nucleic Acids Res 47, D442D450, doi:10.1093/nar/gky1106 (2019).

74 Cox, J. \& Mann, M. MaxQuant enables high peptide identification rates, individualized p.p.b.-range mass accuracies and proteome-wide protein quantification. Nat Biotechno/ 26, 1367-1372, doi:10.1038/nbt.1511 (2008). 
75 Cox, J. et al. Andromeda: A Peptide Search Engine Integrated into the MaxQuant Environment. Journal of Proteome Research 10, 1794-1805, doi:10.1021/pr101065j (2011).

96576 Nolte, H., MacVicar, T. D., Tellkamp, F. \& Kruger, M. Instant Clue: A Software Suite for Interactive Data Visualization and Analysis. Sci Rep 8, 12648, doi:10.1038/s41598-018-31154-6 (2018).

96877 Tyanova, S. et al. The Perseus computational platform for comprehensive 969 analysis of (prote)omics data. Nat Methods 13, 731-740, doi:10.1038/nmeth.3901 (2016).

97178 Cox, J. \& Mann, M. 1D and 2D annotation enrichment: a statistical method integrating quantitative proteomics with complementary high-throughput data. BMC Bioinformatics 13 Suppl 16, S12, doi:10.1186/1471-2105-13-S16-S12 (2012). 

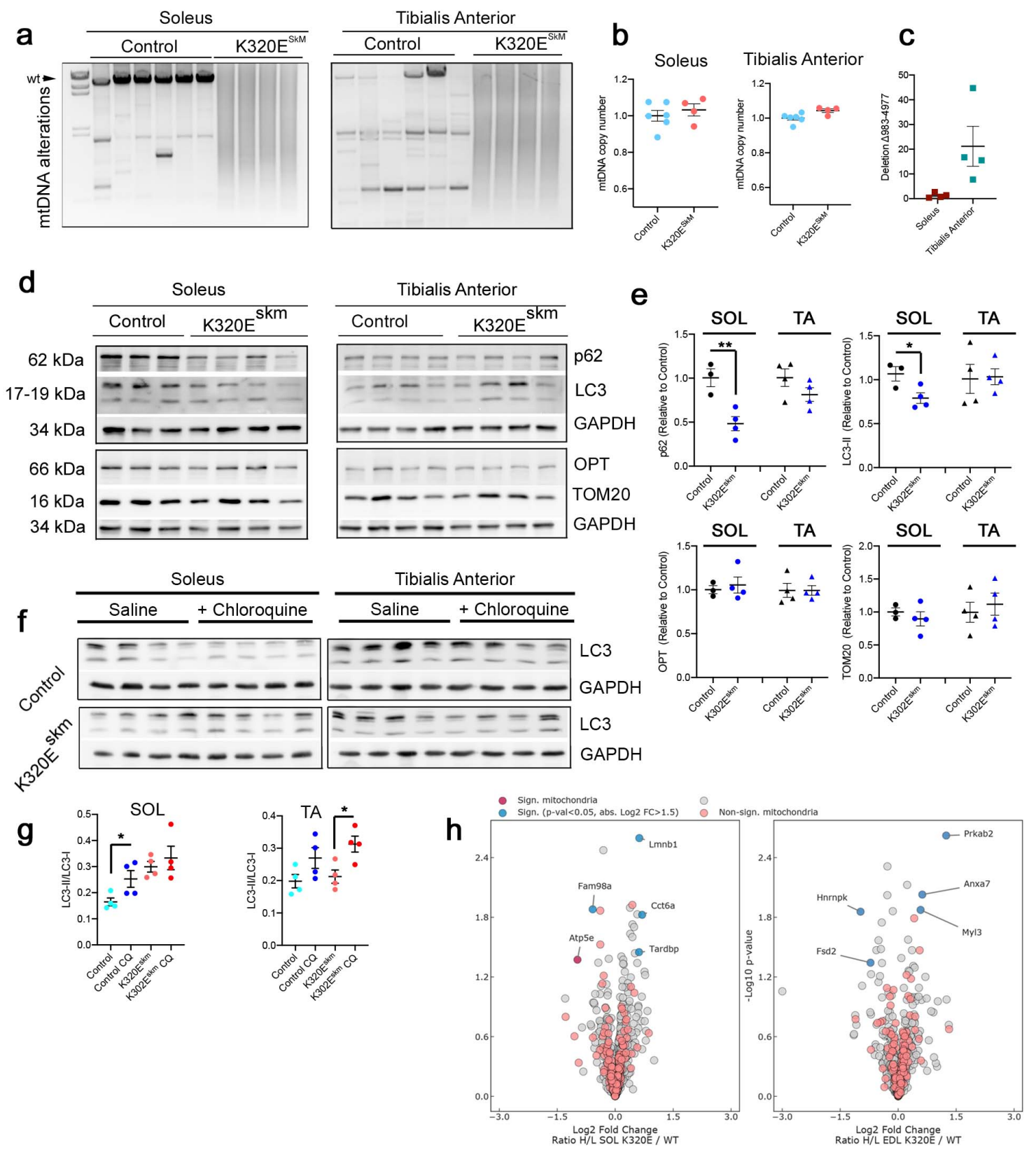

Figure 1. In vivo expression of Twinkle-K320E induces differential accumulation of $m$ tDNA alterations in muscles. (a) Long range PCR analysis, (b) quantification of mtDNA copy number and, (c) qPCR quantification of deletion mtDNA- $\triangle 983-$ 4977 in M. soleus and M. Tibialis anterior from 24 months old control and K320E $\mathrm{E}^{\mathrm{SkM}}$ mice. (d, e) Western blot analysis and quantification of the indicated proteins in muscle extracts from 24 months old mice. M. soleus, control: $n=3$; Twinkle-K320E: $n=4$. M. tibialis anterior, control: $n=4$; Twinkle-K320E: $n=4$. (f, g) Western blot analysis and quantification of LC3 flux (LC3II/LC3-I ratio) in muscle extracts from mice treated with saline or $50 \mathrm{mg} / \mathrm{kg}$ chloroquine 4 hours before euthanasia. $\mathrm{n}=4$ mice per treatment and genotype. Unpaired Students' T-test. Mean \pm SEM. (h) Volcano plots for mitochondrial and nonmitochondrial proteins detected in the muscles analysed for in vivo Pulse SILAC. Red dots: mitochondrial proteins; dark red: mitochondrial proteins with significant difference; blue dots: non mitochondrial proteins with significance difference. ( $p$-value $<0.05$ and $\mathrm{H} / \mathrm{L}$ ratio fold change $>1.5$ ). $\mathrm{n}=5$. 
a

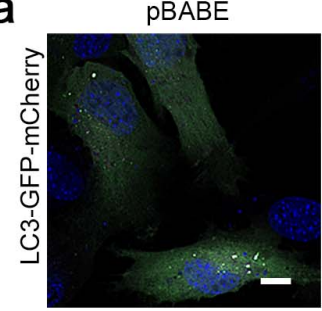

C

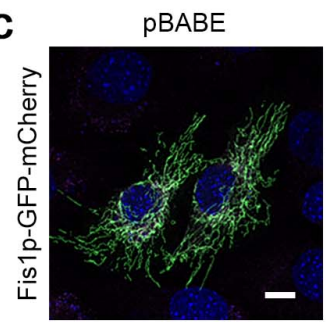

g Twinkle-Cherry

g
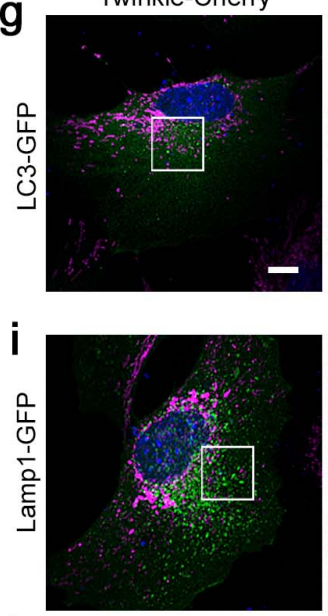

k

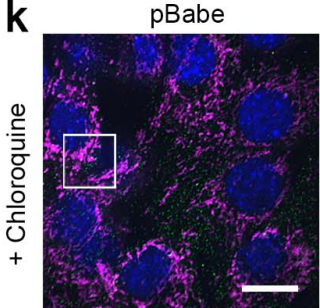

Twinkle

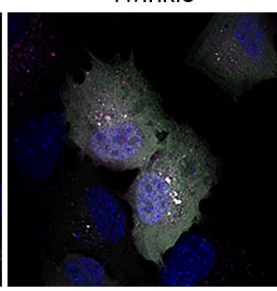

Twinkle

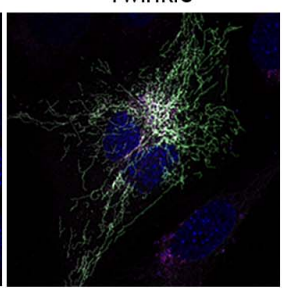

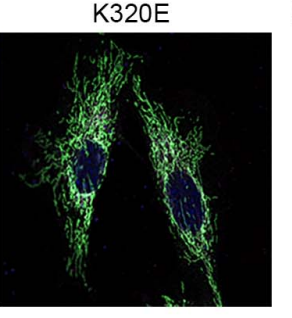

K320E

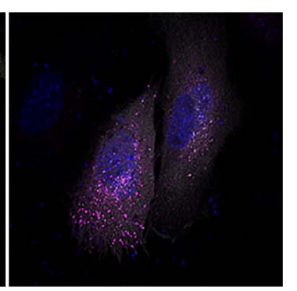

K320E

K320E-Cherry
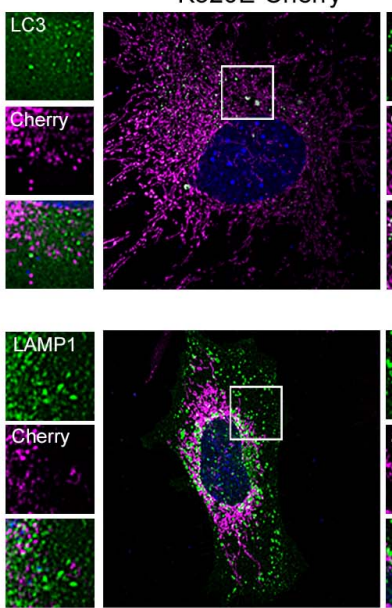

Twinkle

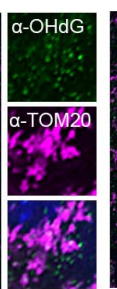

h

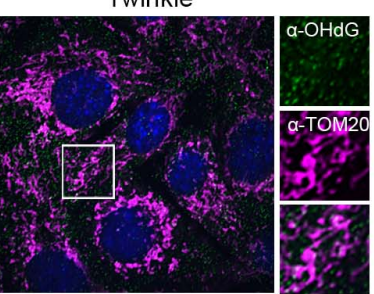

b

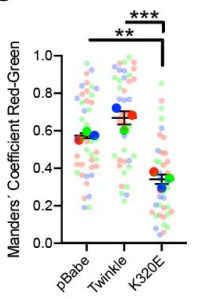

d

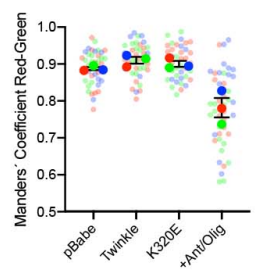

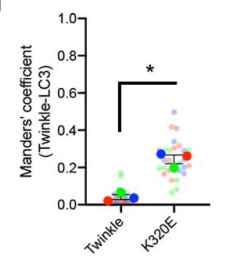
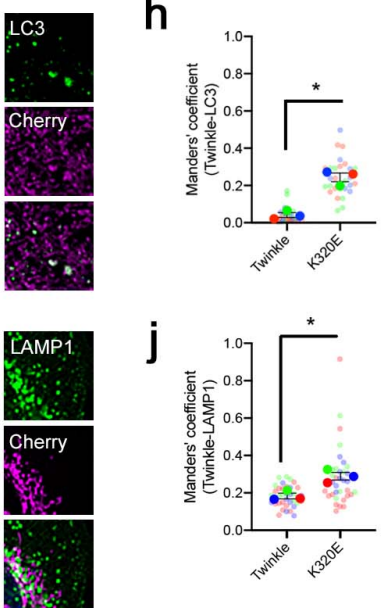

K320E

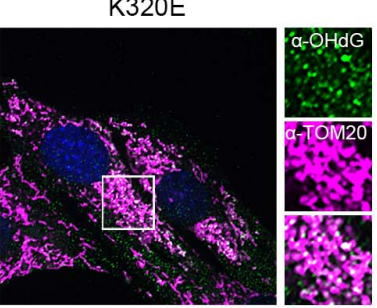

e

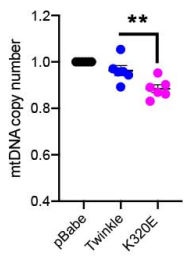

f

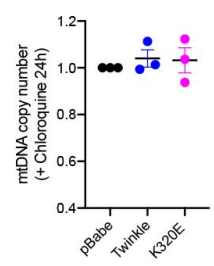

Figure 2. Twinkle-K320E triggers mtDNA damage and induces autolysosome accumulation independent of bulk mitophagy. (a-e) C2C12 expressing untagged Twinkle constructs transiently expressing the autophagy reporter LC3-GFPmCherry or (c) the mitophagy reporter Fis1p-GFP-mCherry. Red signal shows lysosomal localization. (b, c) Manders' coefficient Red / Green quantification of transfected cells. $n=3$. A decrease in Manders' coefficient indicates autolysosome or mito-lysosome accumulation, $n=3,10-15$ cells per replicate. Cells treated overnight with 10 $\mu \mathrm{M}$ Antimycin/Oligomycin were used to induce canonical mitophagy. (e, f) mtDNA copy number in $\mathrm{C} 2 \mathrm{C} 12$ cells stably expressing Twinkle or Twinkle-K320E (K320E), respectively, vs. empty vector (pBABE). In (f), mtDNA levels were recovered by treating cells with the lysosomal inhibitor chloroquine for $24 \mathrm{~h}, \mathrm{n}=3$. ( $\mathbf{g}-\mathbf{h}$ ) Confocal images and quantification of $\mathrm{C} 2 \mathrm{C} 12$ expressing mCherry tagged Twinkle transfected with plasmids encoding the autophagosome marker LC3-GFP and (i, j) lysosome marker Lamp1-GFP. Manders' colocalization coefficient was used to confirm Twinkle colocalization with autophagic organelles, $n=3$ ( $>10$ cells per experiment). (k) mtDNA oxidative damage detected by immunofluorescent labelling with $\alpha-O H d G$ and $\alpha-T O M 20$ in cells expressing Twinkle and treated with chloroquine (CQ) for $24 \mathrm{~h}$. (I) Relative intensity quantification of $\alpha-O H d G$ signal inside the mitochondrial network. Scale Bar, $10 \mu \mathrm{m}$. (c-f and I) ANOVA, Tukey multiple comparison. ${ }^{* *}, p<0.01 ;{ }^{* * *}, p<0.001$. (h and j) Unpaired Student's T-test. *, $p<0.05$. Mean \pm SEM. 

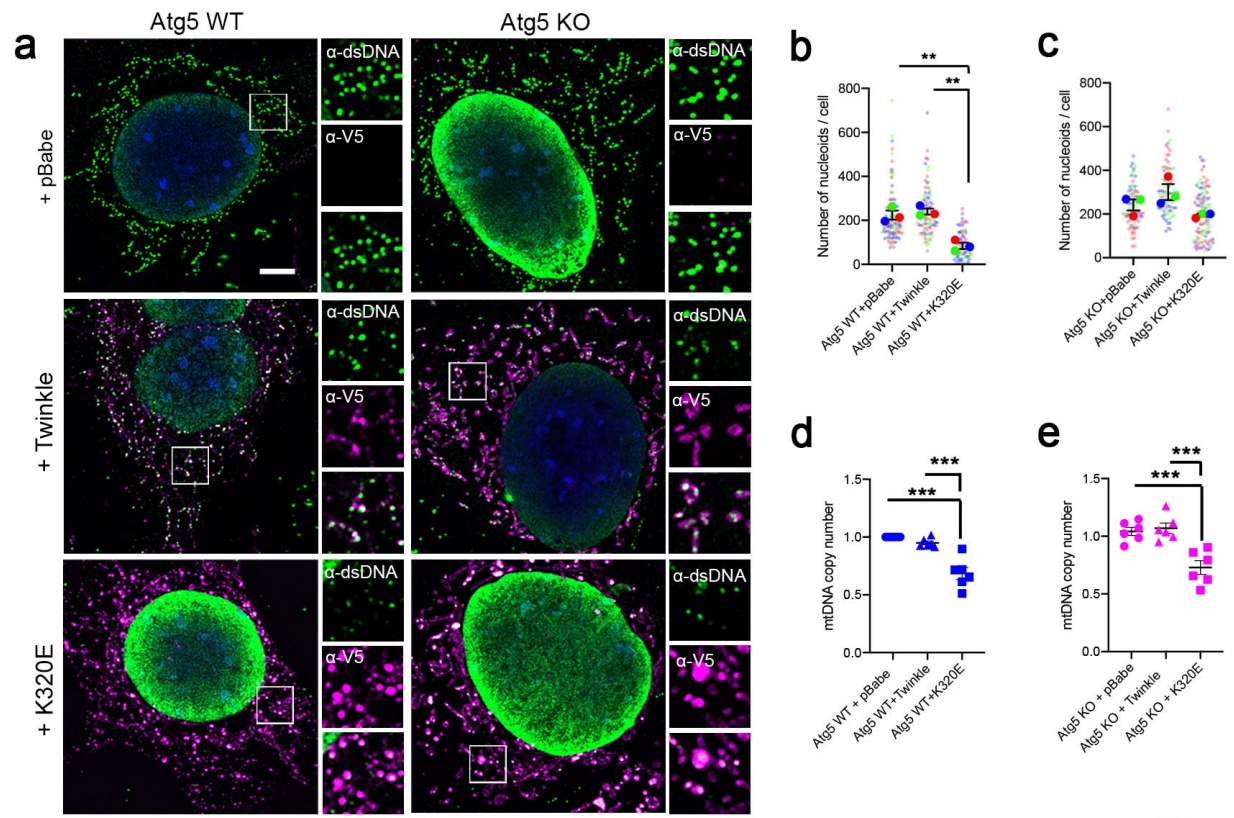

d
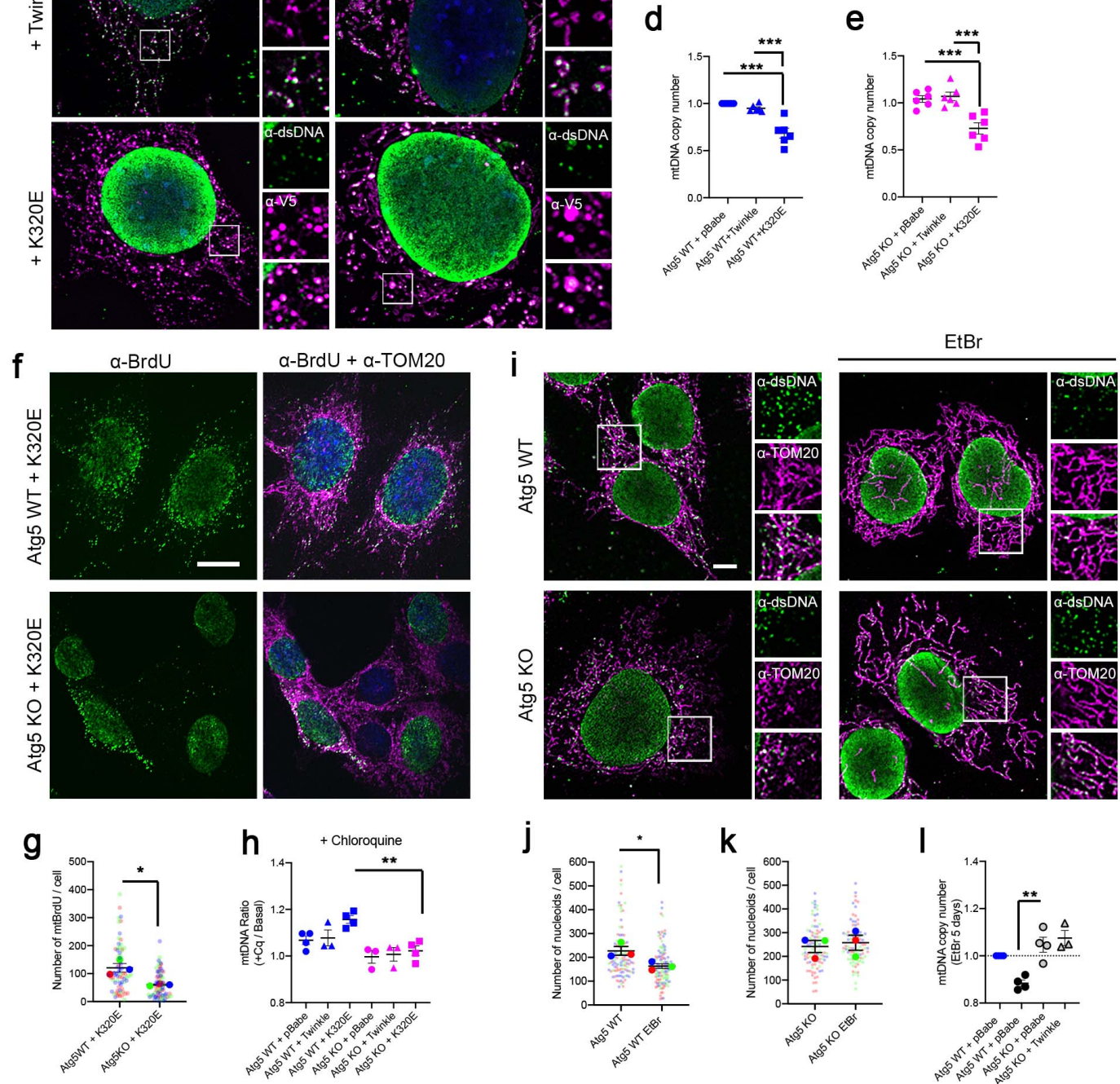

Figure 3. Autophagy is required for mtDNA clearance after mtDNA damage. Atg 5 WT and KO cells were transduced with Twinkle-APEX-V5 plasmids. (a) $\alpha-D N A$ and $\alpha-V 5$-tag immunofluorescence confirming localization of Twinkle in mtDNA containing nucleoids. Scale bar $5 \mu \mathrm{m}$. (b, c) Quantification of mtDNA foci number in Atg5 WT and Atg5 KO cells. $\mathrm{n}=3$ (>25 cells per experiment). (d, e) Steady state mtDNA copy number in Atg 5 cells. (f, g) $\alpha$-BrdU and $\alpha$-TOM20 immunofluorescence and quantification of mtDNA replicating foci detected by treating the cells for $6 \mathrm{~h}$ with $20 \mu \mathrm{M}$ BrdU. $n=3,>20$ cells per replicate (h) Quantification of mtDNA copy number ratio in Atg 5 cells treated with Chloroquine for $24 \mathrm{~h} . \mathrm{n}=3$. (i-k) $\alpha$-DNA and $\alpha$-TOM20 immunofluorescence and mtDNA foci quantification in steady state and in cells treated with $50 \mathrm{ng} / \mathrm{ul} \mathrm{EtBr}$ for 7 days. $n=3,>20$ cells per replicate. (I) mtDNA copy number analysis for steady state and in cells treated for 7 days with EtBr. $n=3-4$. Scale

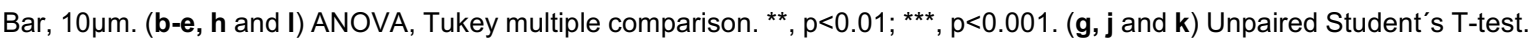
${ }^{*}, p<0.05$. Mean \pm SEM. 
a

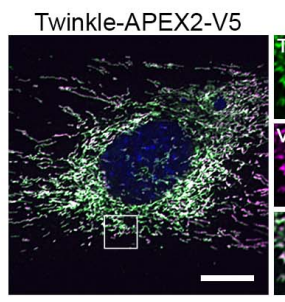

b

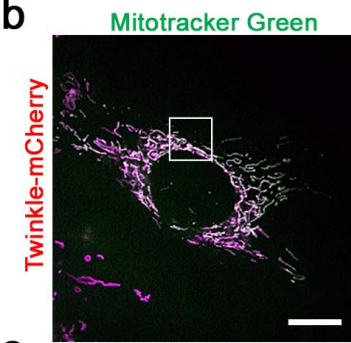

C
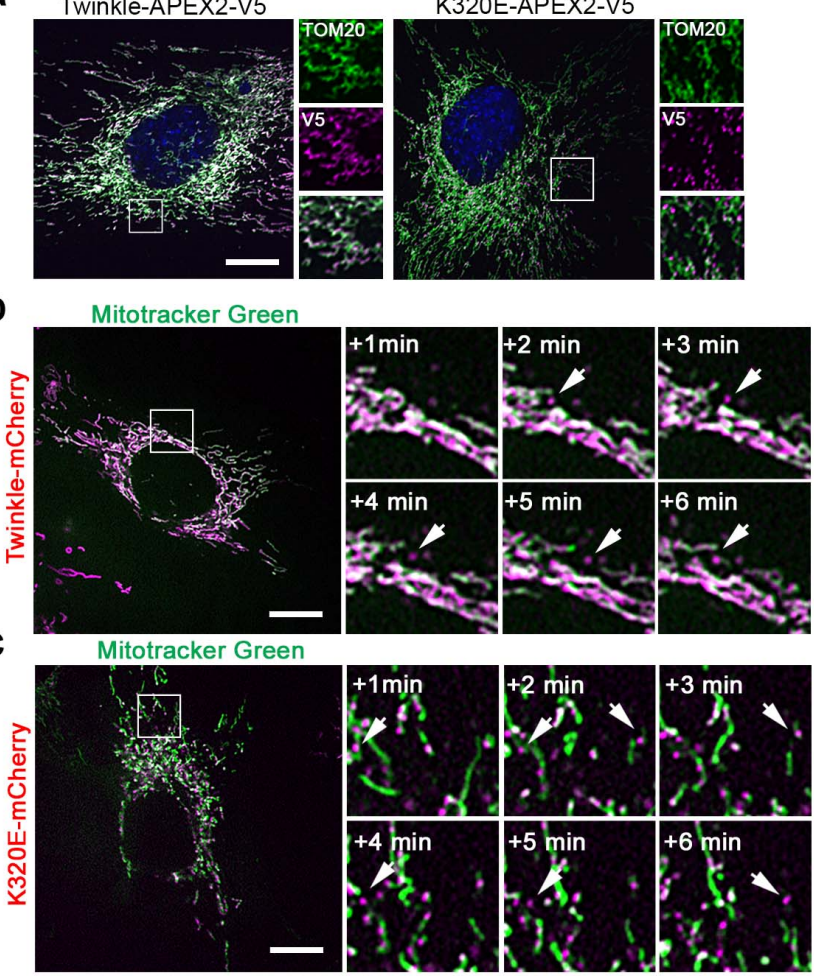

$+1 \mathrm{~min}$ d
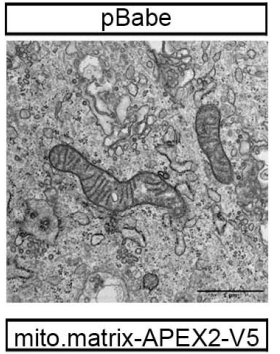

e

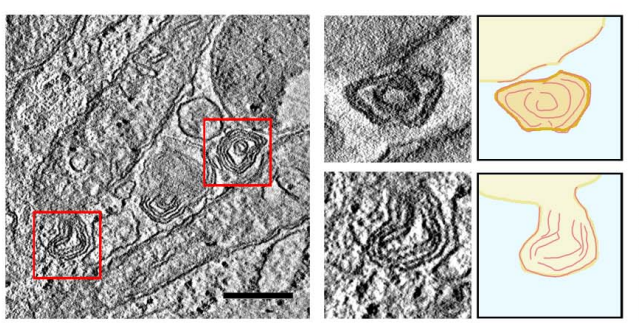

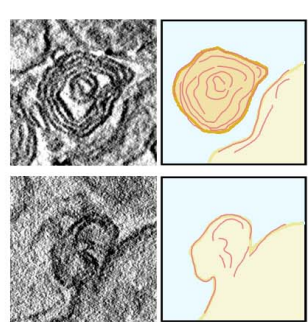

Figure 4. Twinkle-K320E localizes to specialized mitochondrial regions. (a) Immunofluorescence of $\mathrm{C} 2 \mathrm{C} 12$ cells expressing Twinkle-APEX-V5 variants probed with $\alpha-T O M 20$ and $\alpha-V 5$ antibodies. Arrow shows TOM20 negative particle

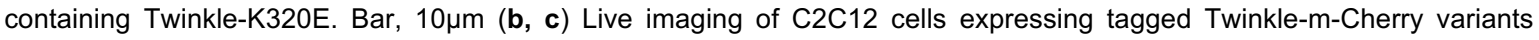
counterstained with mitotracker green. Insets represent a $1 \mathrm{~min}$ time lapse series of the selected area. Bar, 10 $\mu \mathrm{m}$ (d) Transmission electron microscopic pictures of C2C12 cells expressing APEX2-V5 clones. APEX2 generates a black precipitate in presence of $\mathrm{DAB}$. Red arrows indicate multimembrane structures, asterisks indicate autophagosomes. Bar, $1 \mu \mathrm{m}$.

(e) Electron tomography in $\mathrm{C} 2 \mathrm{C} 12$ cells expressing Twinkle-K320E illustrating extrusion of mitochondrial fragments containing multimembrane structures. Bar, $0,5 \mu \mathrm{m}$ 

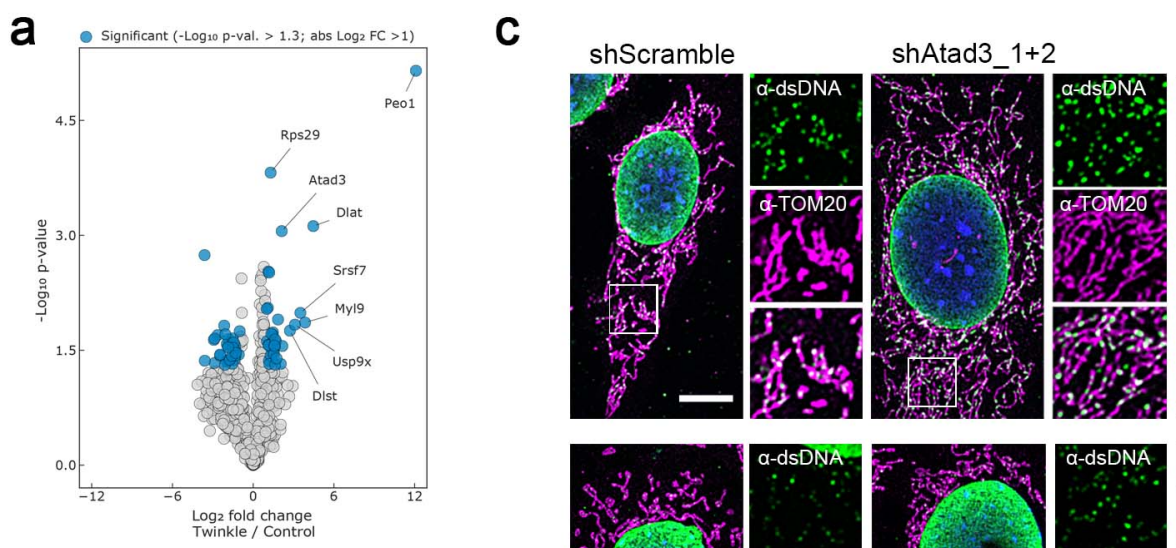

shAtad3_3
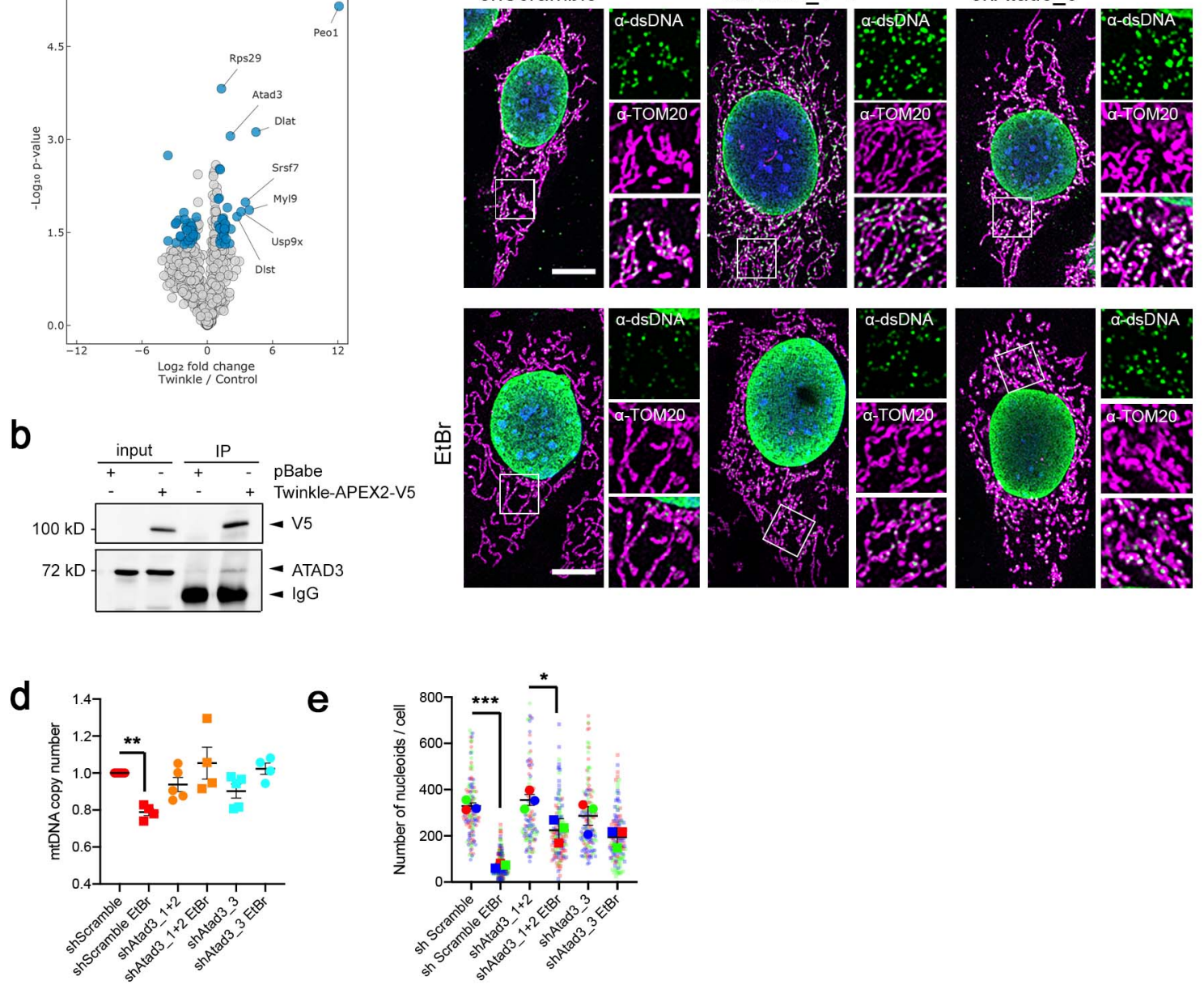

Figure 5. Twinkle mediates interaction of nucleoids with the inner mitochondrial membrane protein ATAD3. (a) Volcano plot showing proteins enriched after immunoprecipitation of Twinkle-APEX2-V5 in C2C12 cells. Differentially enriched proteins compared with cells transfected with empty vector ( $p$-value $<-0.05$ and $F C>2$ or $<-2$ ) are highlighted in blue (Peo1 = Twinkle). (b) Co-immunoprecipitation of V5-tagged Twinkle and ATAD3. (c) $\alpha$-TOM20 and $\alpha$-dsDNA immunofluorescence in Atad3 KD MEFs in steady state and grown for 7 days in presence of $50 \mathrm{ng} / \mathrm{ml} \mathrm{EtBr}$. Bar, $10 \mu \mathrm{m}$. (d) Quantification of mtDNA copy number in steady state and $\mathrm{EtBr}$ treated cells. $n=4-5$. and (e) mtDNA foci quantification for Atad3 KD in steady state and EtBr treated cells. $n=3,>20$ cells per replicate. ANOVA, Tukey multiple comparison. ${ }^{*}, p<0.05,{ }^{* *}, p<0.01$. Mean \pm SEM. 
a
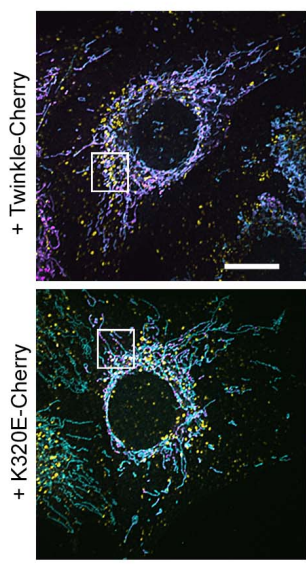

C

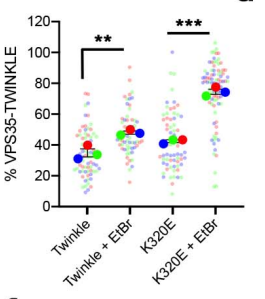

f

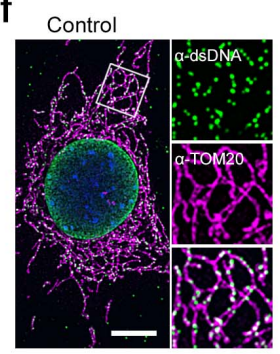

i

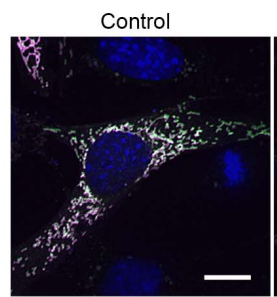

d
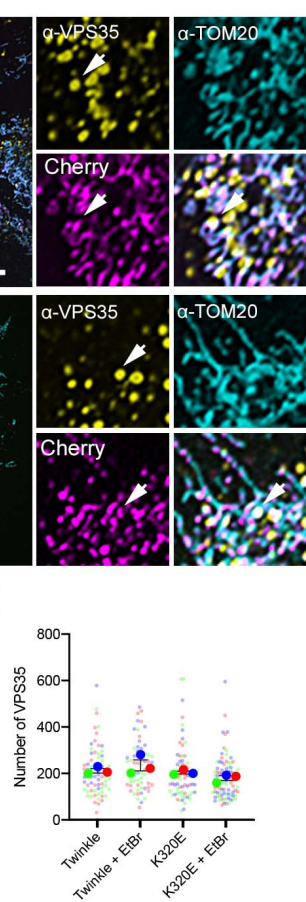

Vps35 Ex4 KO

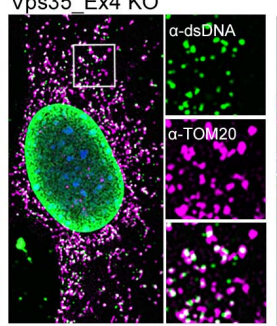

Vps35_Ex4

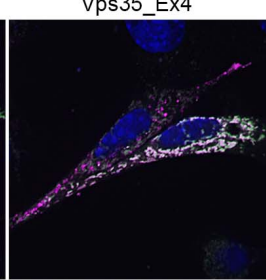

b
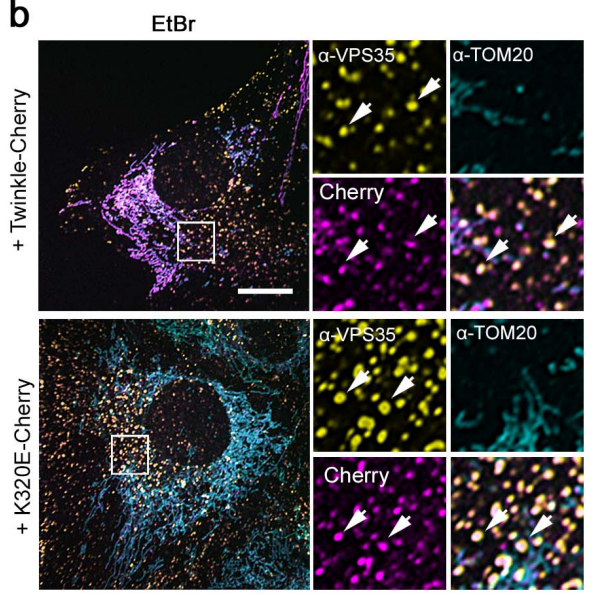

e

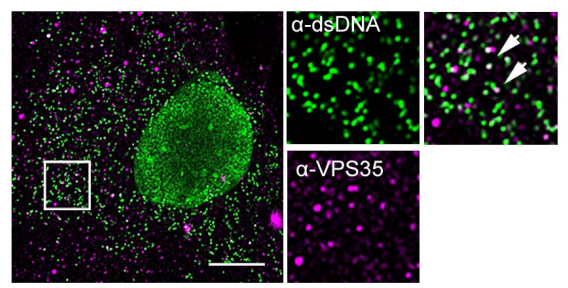

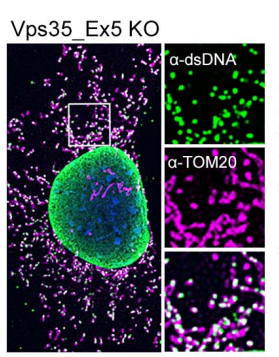

g
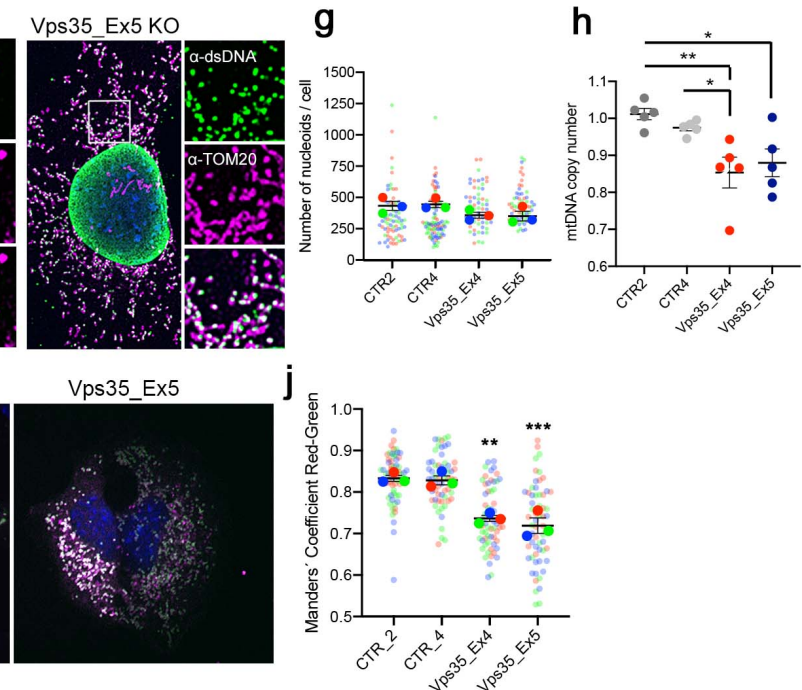

Figure 6. VPS35 is required for autophagy-dependent mtDNA removal. Immunofluorescence of C2C12 cells expressing Twinkle-mCherry variants labelled with $\alpha$-VPS35 and $\alpha$-TOM20 and grown in (a) basal medium or (b) treated for 7 days with $50 \mathrm{ng} / \mathrm{ml}$ EtBr. Arrows indicate colocalization. Bar, 10 $\mu \mathrm{m}$. (c, d) Quantification of VPS35 particles in contact with Twinkle. $\mathrm{n}=3$ (e) Immunofluorescence of C2C12 wild type cells labelled with a-dsDNA and a-VPS35. Arrows indicate colocalization between

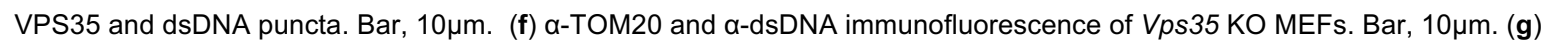
mtDNA foci analysis $(n=3)$ and $(\mathbf{h})$ mtDNA copy number quantification of Vps35 KO cells. $n=5$. (i) Control and Vps35 KO cells transfected with Fis1p-GFP-mCherry plasmid to detect mitophagy. Red signal represents mito-lysosomes. Bar, 10um. (j) Manders' coefficient quantification of transfected cells. $n=3$. A decrease in Manders' coefficient indicates process activation. $\mathrm{n}=3$ (20 images per replicate). ANOVA, Tukey multiple comparison. ${ }^{*}, \mathrm{p}<0.05 ;{ }^{* *}, \mathrm{p}<0.01 ;{ }^{* * *}, \mathrm{p}<0.001$. Mean $\pm \mathrm{SEM}$ 


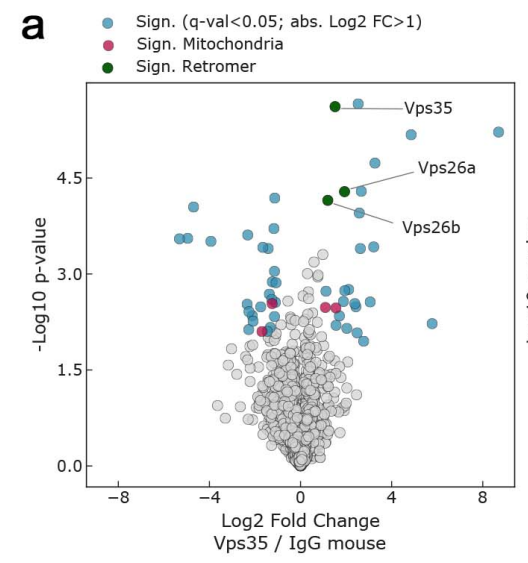

b

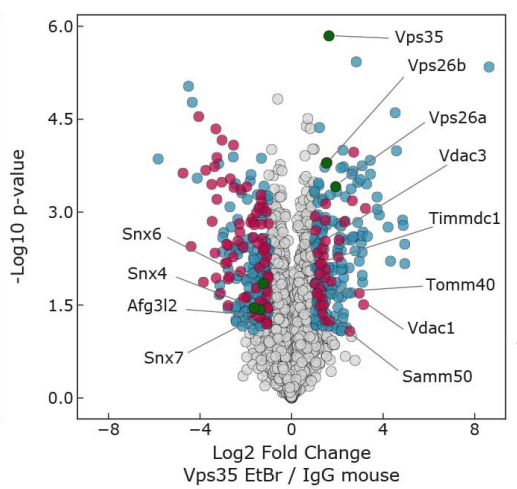

C

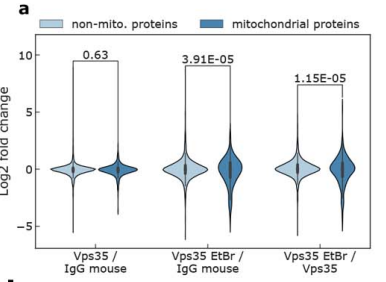

d $\frac{\text { input }}{+} \frac{\text { control }}{+} \frac{\mathbb{P}}{+}$ EtBr

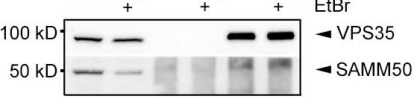

e
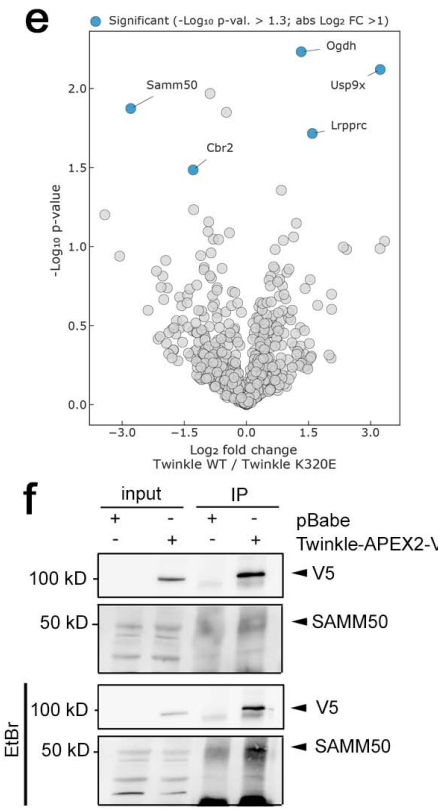

g
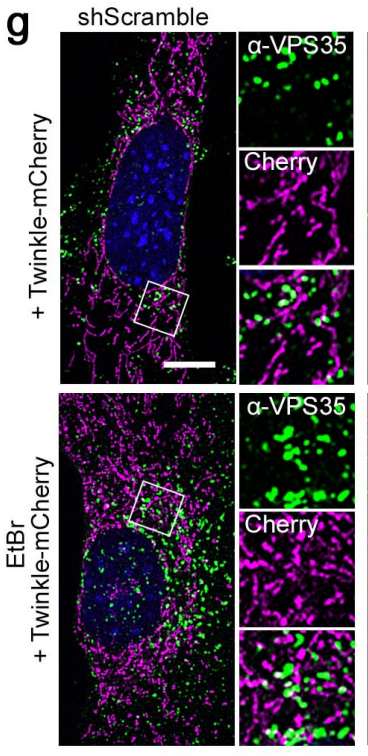

shSamm50 KD $\mathrm{h}$

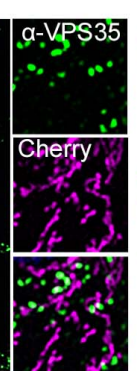

shSamm50 KD
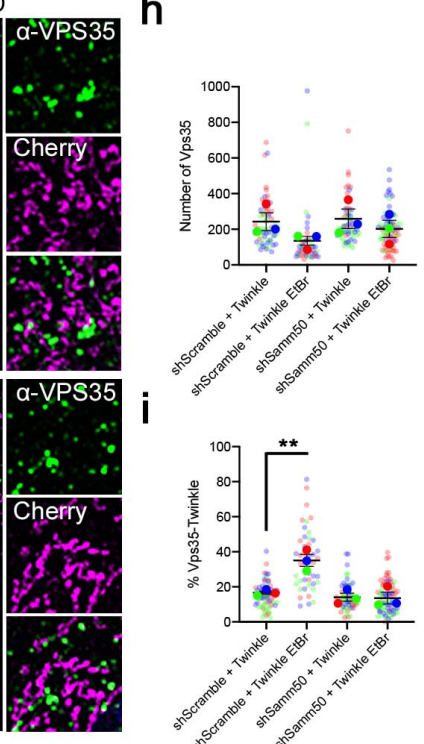

i

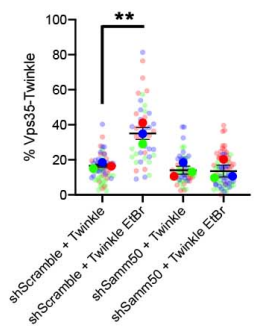

Figure 7. SAMM50 is a mitochondrial receptor for VPS35 recruitment into mitochondrial subcompartments containing nucleoids. (a) Volcano plot showing proteins enriched after immunoprecipitation of VPS35 in MEFs. Only differentially enriched proteins are highlighted (adj. p-value $<0.05$ and FC $>2$ or $<-2$ ). Blue, non-mitochondrial proteins; Red, mitochondrial proteins; Green, retromer proteins (b) Comparison of VPS35 IP profiles of cells grown in normal medium or 1 week treated with $50 \mathrm{ng} / \mathrm{ml} E t B r . n=3$. (c) Violin Plots showing enrichment of mitochondrial proteins upon pull down of VPS35 in presence of EtBr. Numbers indicate p-value. Students' T-test. (d) Co-Immunoprecipitation of VPS35 and SAMM50 in steady state and $\mathrm{EtBr}$ treated cells. IgG from mouse was used as a control. (e) Comparison of interactome profiles of Twinkle and K320E. $n=3$. (f) co-Immunoprecipitation of Twinkle and SAMM50 in steady state and in cells treated with EtBr for 1 week. (g) Immunofluorescence of control and Samm50 KD MEFs transduced with Twinkle-mCherry constructs and labelled with $\alpha$ VPS35 in basal and 7 days treated with $50 \mathrm{ng} / \mathrm{ml}$ EtBr. Bar, 10 $\mu \mathrm{m}$. (h, i) Quantification of VPS35 particles and VPS35 in contact with Twinkle. $n=3,>20$ cells per replicate. ANOVA, Tukey multiple comparison. ${ }^{* *}, p<0.01$. Mean \pm SEM 
a
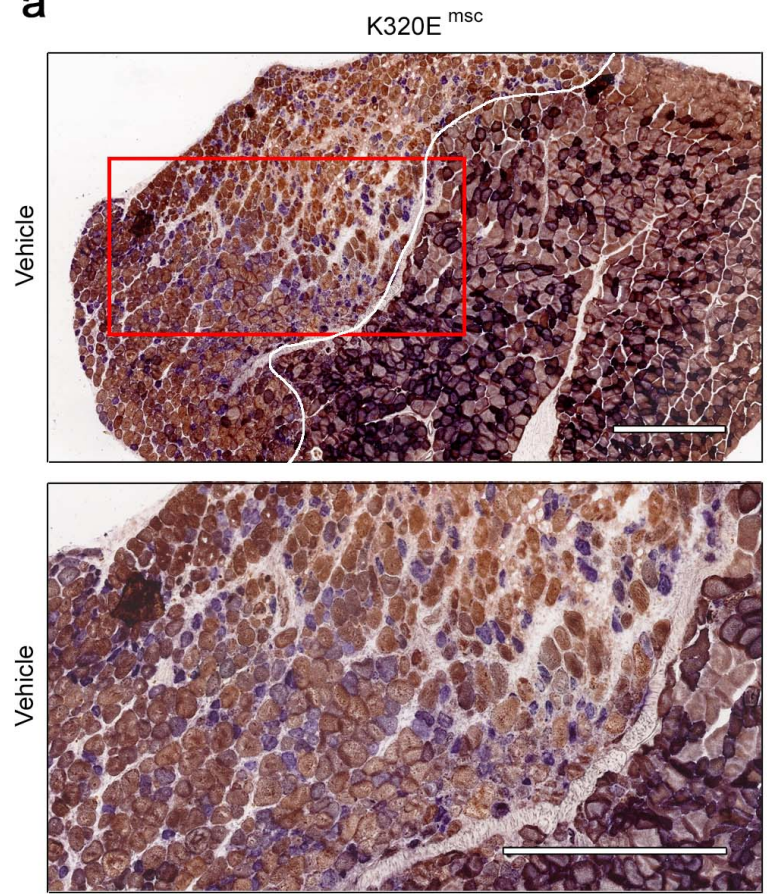

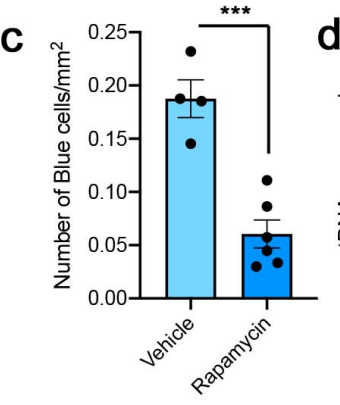

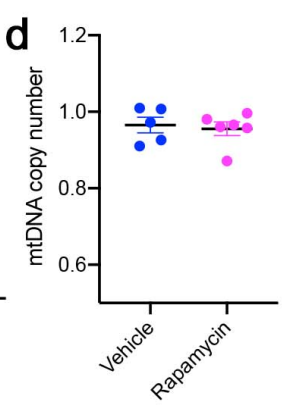

b
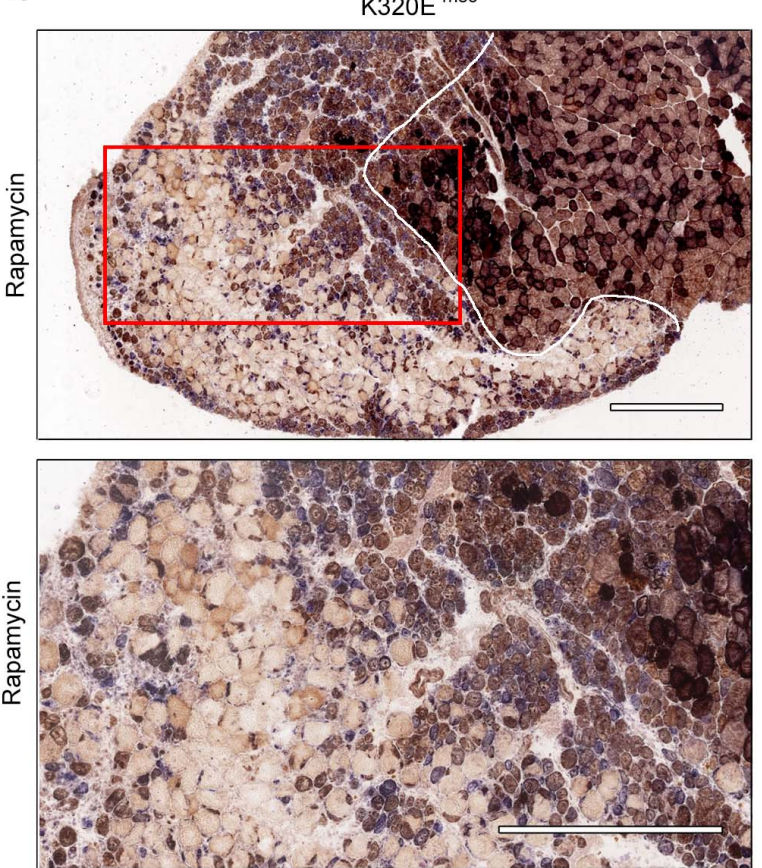

e

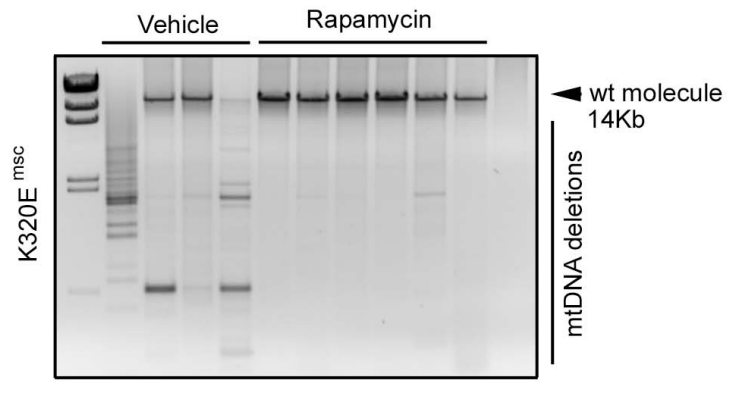

Figure 8. Rapamycin eliminates mtDNA deletions without affecting copy number in vivo. COX-SDH staining of

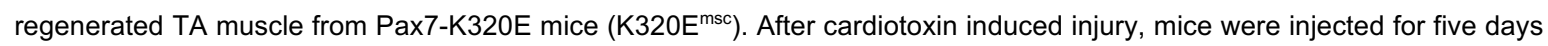

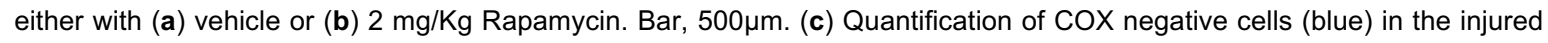
area. (d) mtDNA quantification by qPCR or (e) Long-range PCR in regenerated muscle from K320E ${ }^{\mathrm{msc}}$ mice treated with vehicle or with Rapamycin. n=5-6 mice per condition, genotype and treatment. (c and d) Unpaired Students' T-test. Mean \pm SEM. 

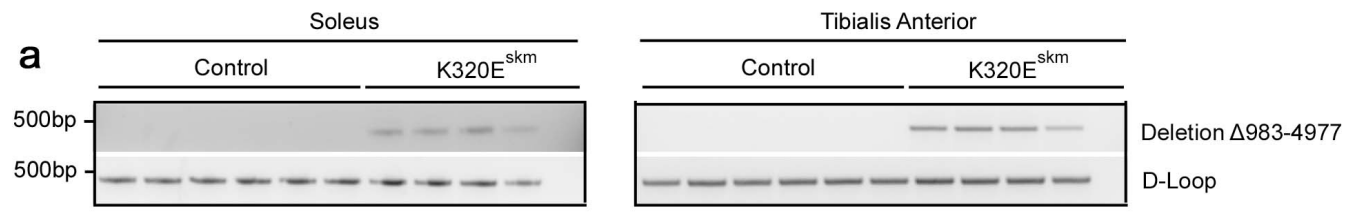

b

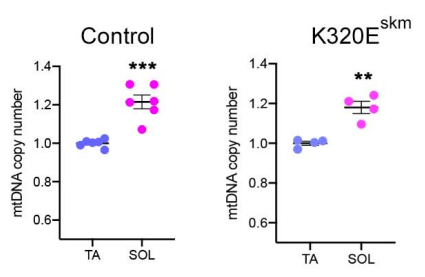

C

C
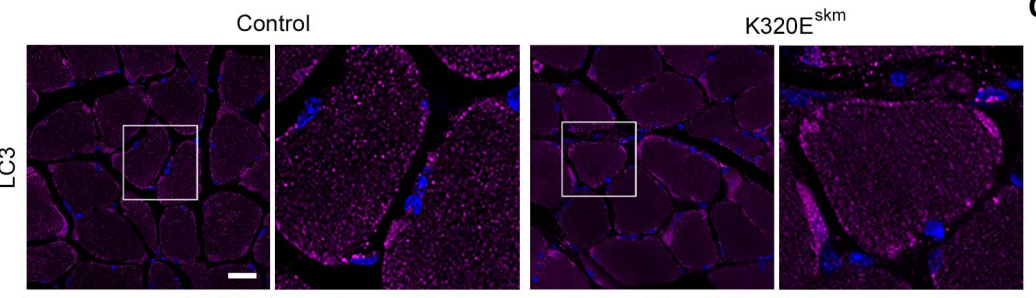

d
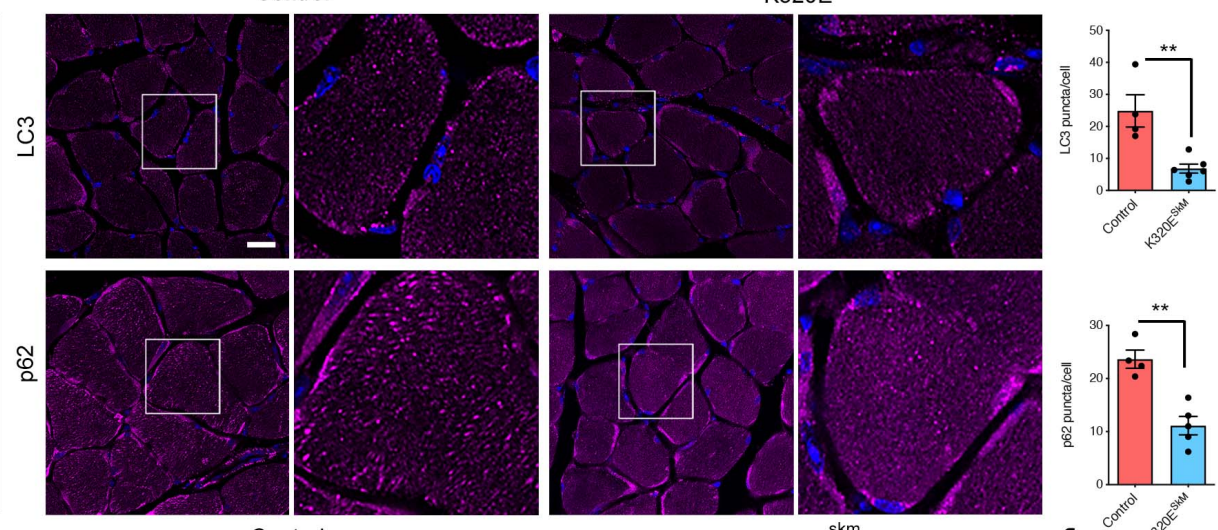

e
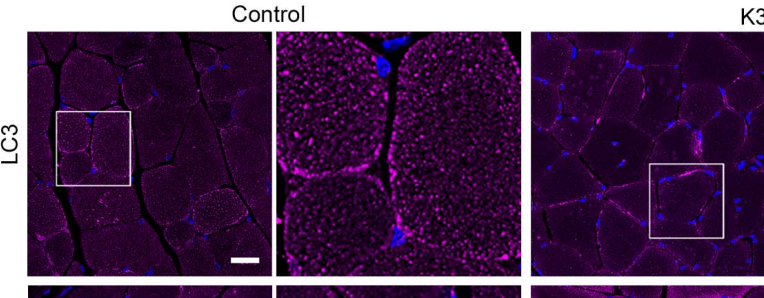

$\mathrm{K}_{320 \mathrm{E}^{\mathrm{skm}}}$
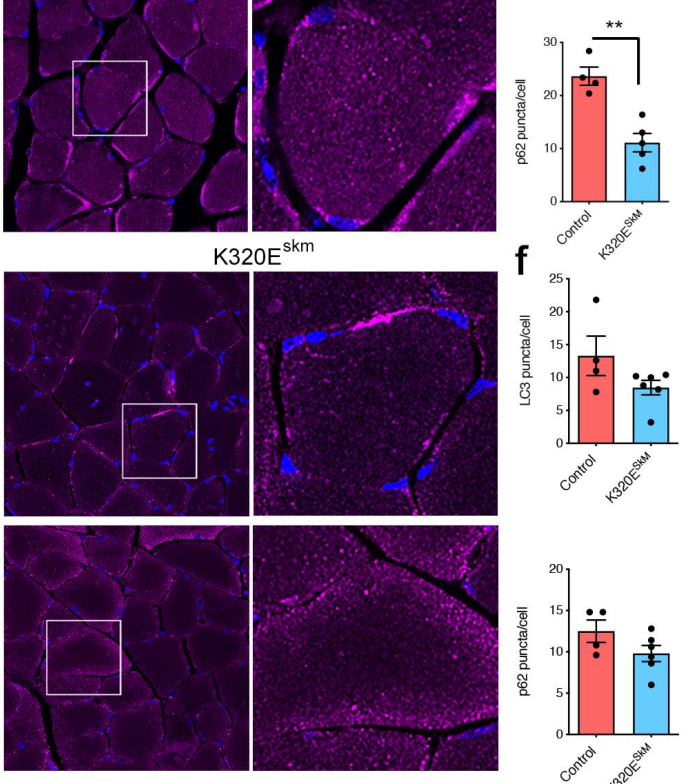

Figure S1. (a) Conventional PCR with specific oligonucleotides flanking the deletion mtDNA- $\triangle 983-4977$. (b) mtDNA copy number in muscles from 24 months old control and Twinkle-K320E mice. This graph shows a comparison between samples analysed in Fig 1b. (c-f) In situ immunofluorescence and image quantification showing autophagic markers LC3 and p62 in cryosections of M. soleus (c, d) and M. tibialis anterior (e, f). 5 random pictures with 4 fibers per picture were analysed per

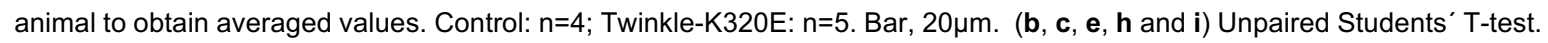
Mean \pm SEM. 
a $\quad \square$ EDL K320E $\square$ SOL K320E
$\square$ EDL WT
SOL WT

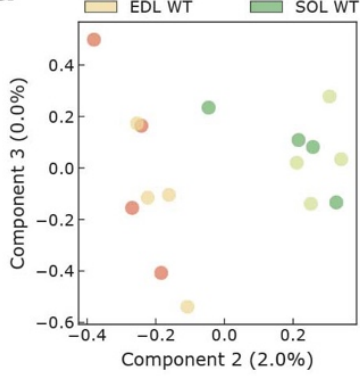

\section{b}
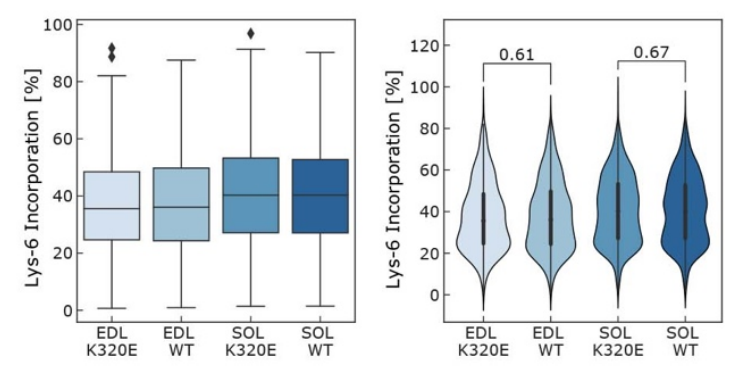

C

MitoCarta3.0

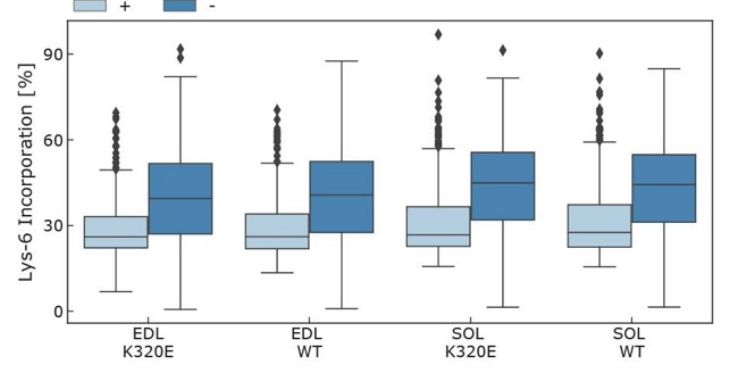

Figure S2. (a) Component analysis of In vivo Pulse SILAC in M. extensor digitorum longus (EDL) and M. soleus. (b) Box plot and Violin plots showing Lys-6 incorporation in muscles from Twinkle mice. (c) Box plot analysis for mitochondrial and nonmitochondrial proteins detected in the muscles analysed for in vivo Pulse SILAC. $n=5$. 

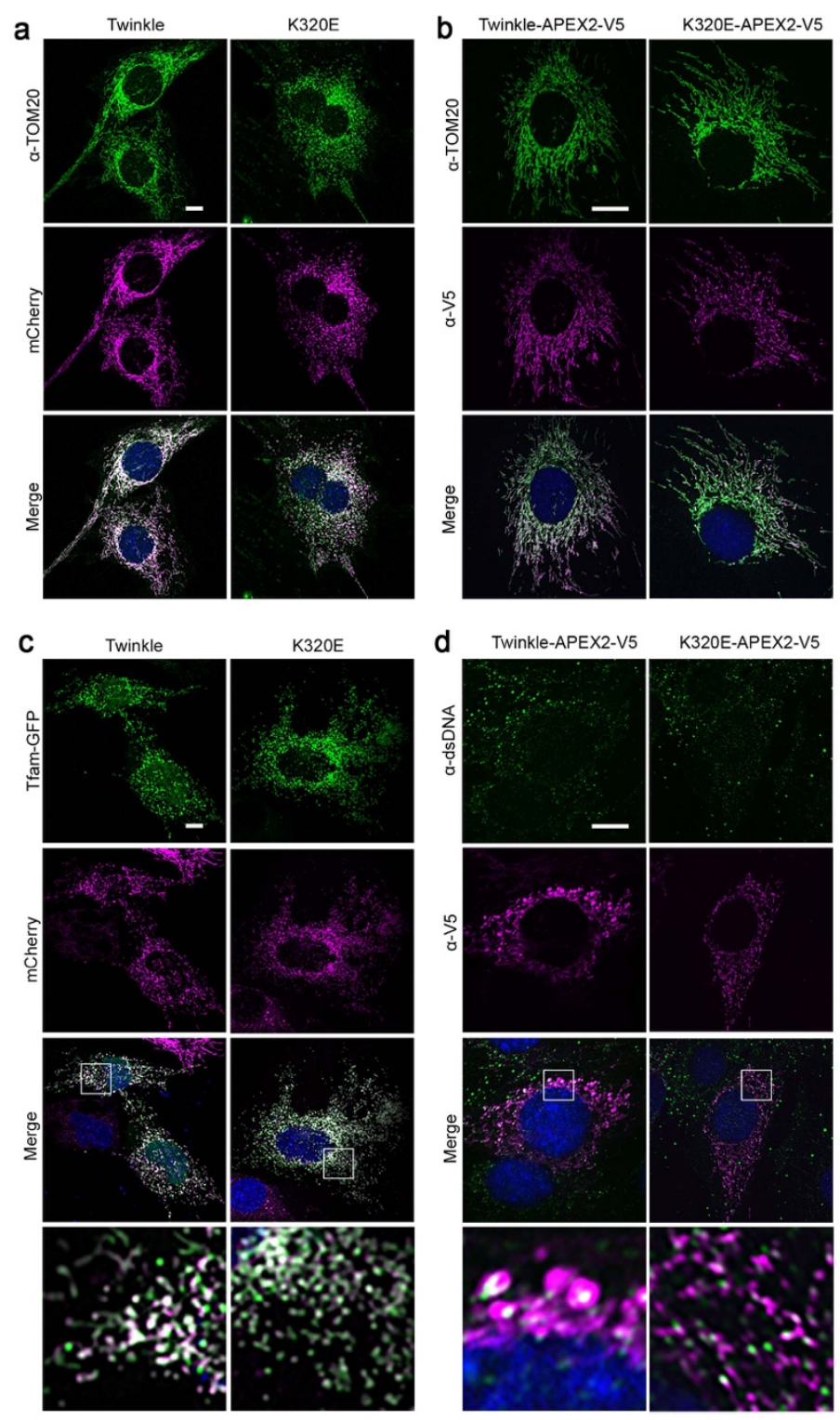

Figure S3. (a, b) $\alpha$-TOM20 immunofluorescence of clones expressing (a) m-Cherry and (b) APEX2-V5 tagged Twinkle variants. (c, d) Immunofluorescence of (c) m-Cherry tagged clones transiently expressing Tfam-GFP and (d) APEX-V5 tagged clones probed with $\alpha$-dsDNA antibody showing nucleoid localization (see magnifications in lower panels). Bar, $10 \mu \mathrm{m}$. 
a

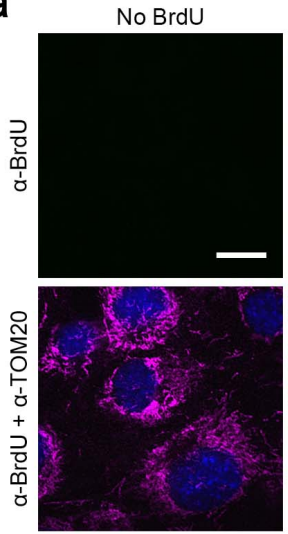

c

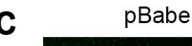

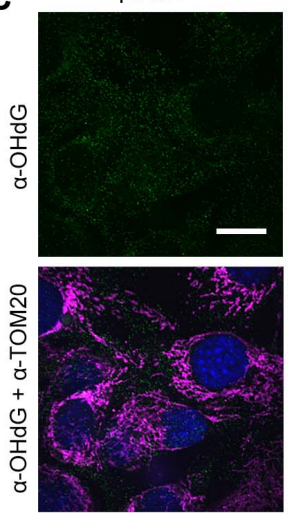

e

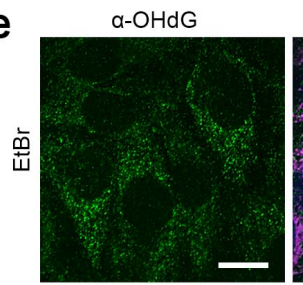

Twinkle

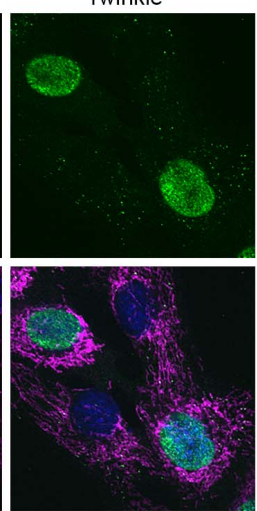

Twinkle

K320E

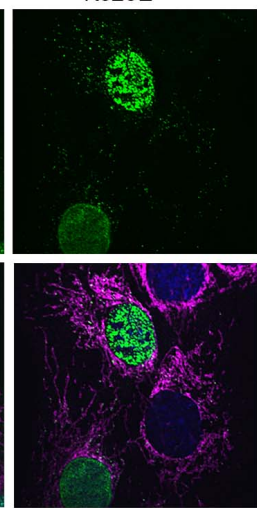

K320E
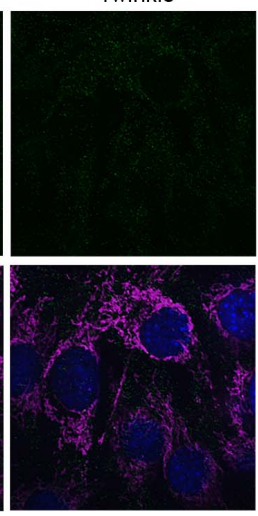

$\alpha-O H d G+\alpha-T O M 20$

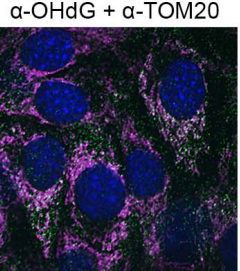

b

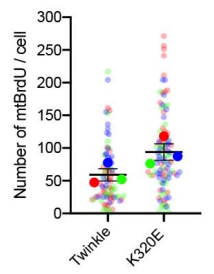

d

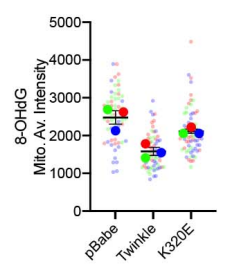

Figure S4. (a, b) Analysis of mtDNA replicating foci by $\alpha$-BrdU and $\alpha$-TOM20 immunofluorescence of C2C12 cells expressing Twinkle plasmids treated with $20 \mu \mathrm{M}$ BrdU. Only BrdU foci inside the mitochondrial network were analysed. (c, d) $\alpha-O H d G$ and $\alpha$-TOM20 immunofluorescence and quantification of the average intensity inside the mitochondrial network of $\mathrm{C} 2 \mathrm{C} 12$ in steady state. $(\mathbf{e}, \mathbf{f}) \alpha-O H d G$ and $\alpha-T O M 20$ immunofluorescence and quantification of the average intensity inside the mitochondrial network in C2C12 treated $24 \mathrm{~h}$ with $50 \mathrm{ng} / \mathrm{ul}$ EtBr. Students' T-test. Mean \pm SEM. *, p<0.05. Scale Bar, $10 \mu \mathrm{m}$. 
a

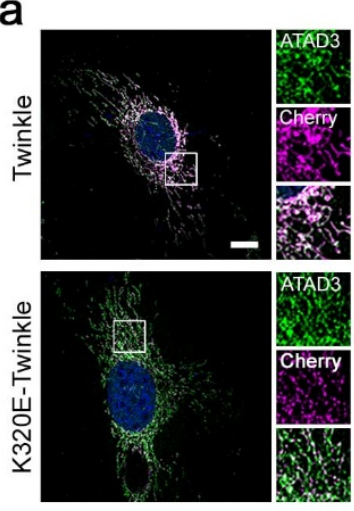

b
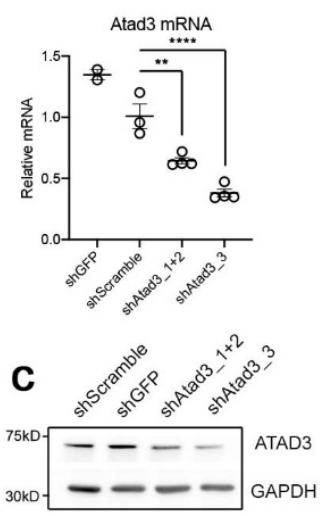

d

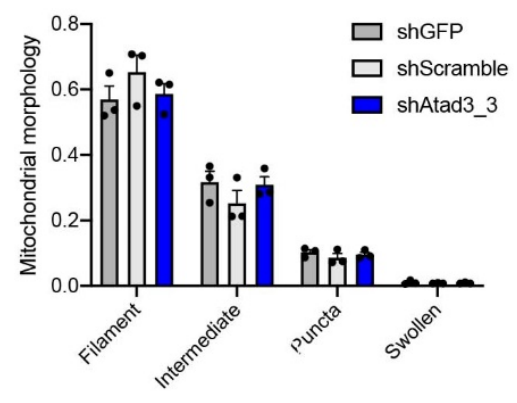

Figure S5. (a) Immunofluorescence of C2C12 cells expressing Twinkle-mCherry stained with a-ATAD3 antibody. Scale bar, $10 \mu \mathrm{m}$. (b) Analysis of Atad3 expression by RT-qPCR and (c) western blot upon shRNA transduction. n=3-4 (d) Quantification of Mitochondrial morphology in shATAD3 cells. $n=3,>30$ cells per replicate. (b and $\mathbf{d}$ ) ANOVA, Tukey multiple comparison. **, $p<0.01 ;{ }^{* * * *}, p<0.001$. Mean \pm SEM. 
a
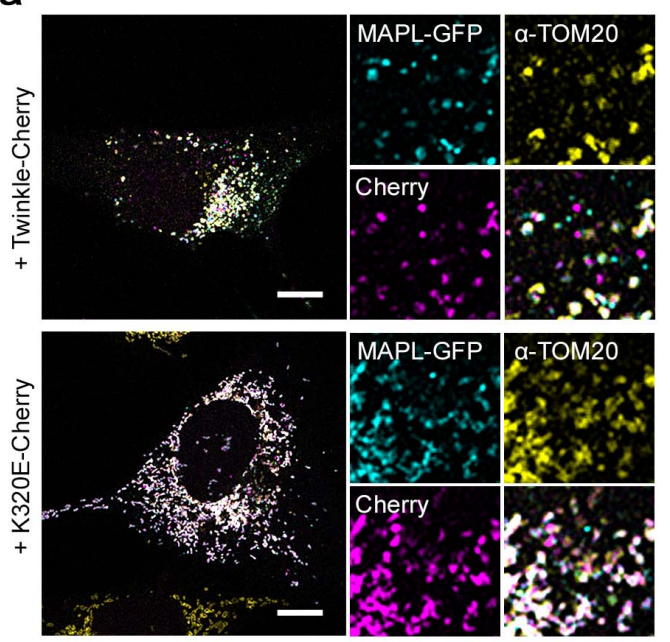

C
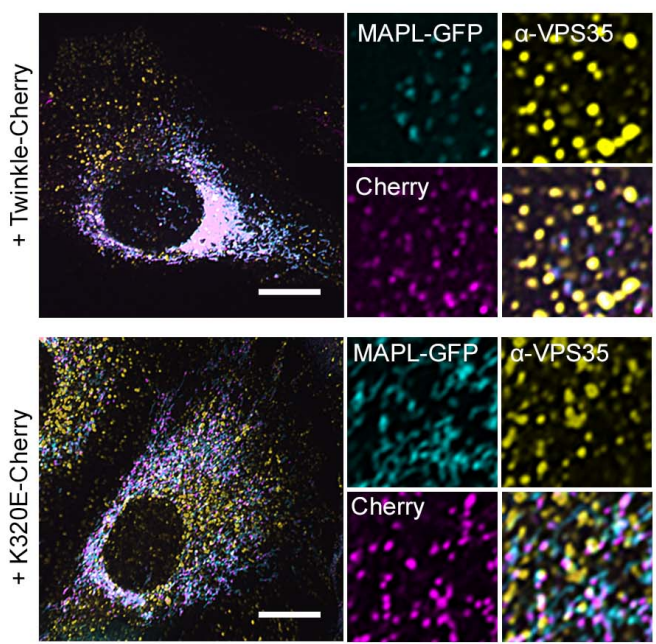

b
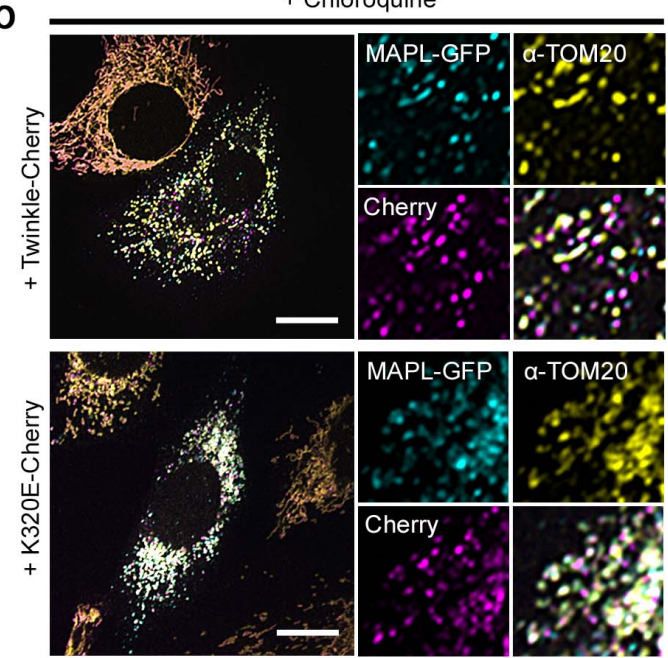

d
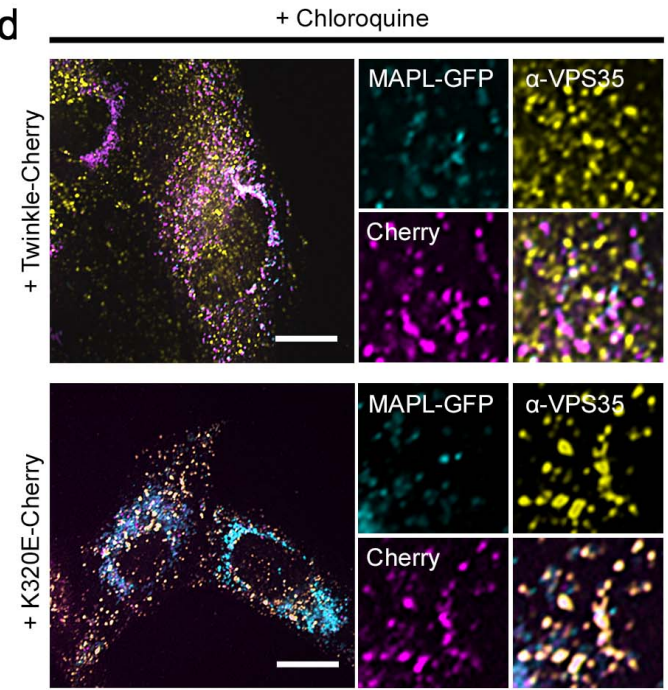

Figure S6. (a-c) Immunofluorescence of $\mathrm{C} 2 \mathrm{C} 12$ cells expressing Twinkle-mCherry transiently transfected with MAPL-GFP and stained with $\alpha$-TOM20 antibody (a, b) or $\alpha-$ VPS35 antibody $(\mathbf{c}, \mathbf{d})$. In (b, d) Cells were treated $4 \mathrm{~h}$ with $10 \mu \mathrm{M}$ Chloroquine. Bar, $10 \mu \mathrm{m}$. 


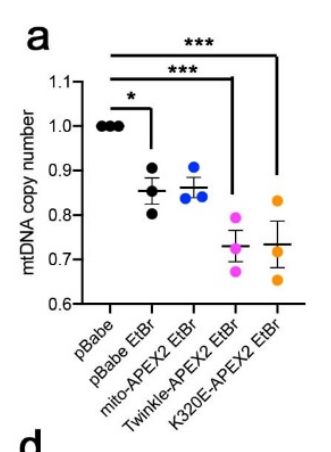

b
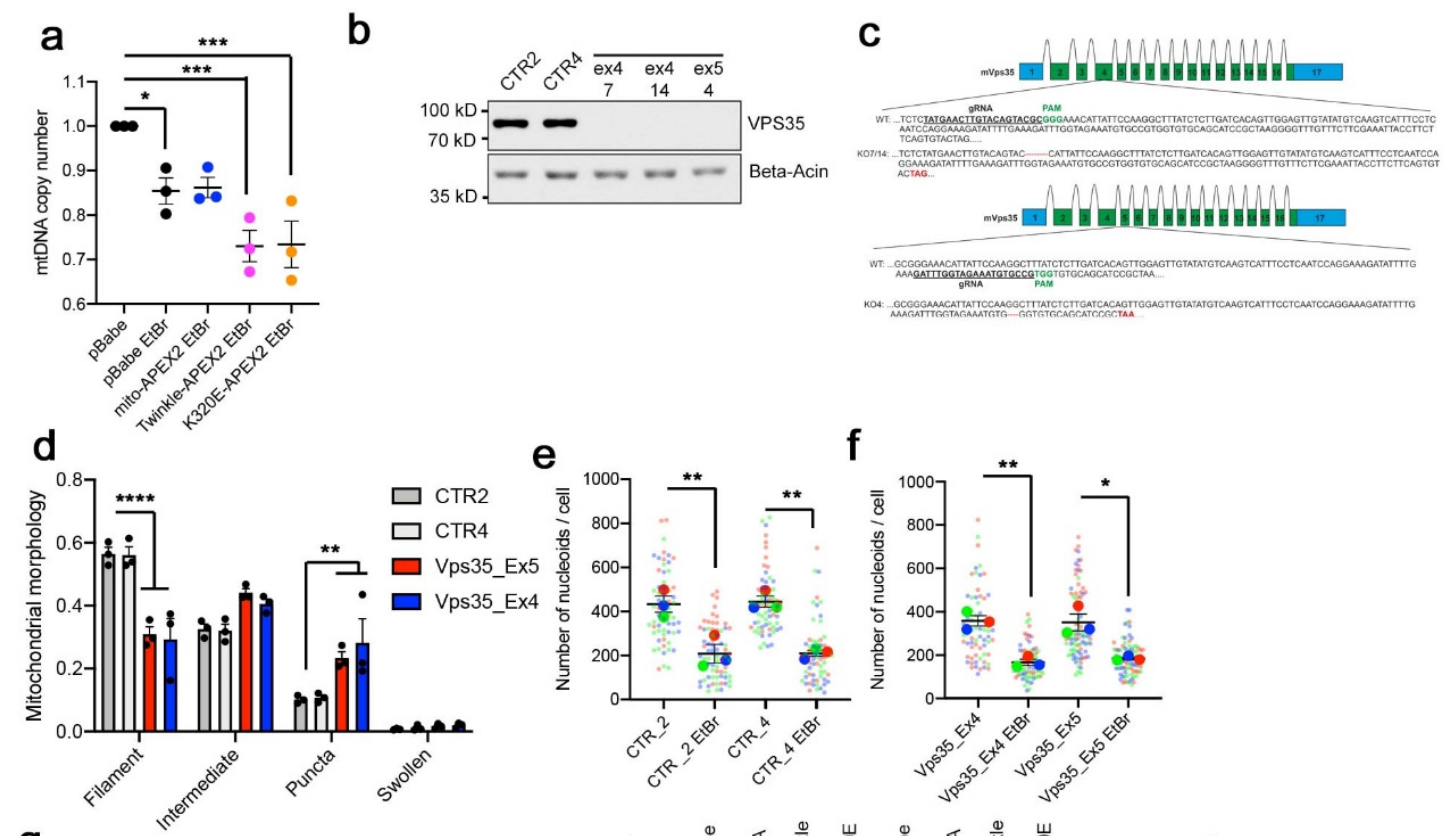

g

$\mathrm{h}$
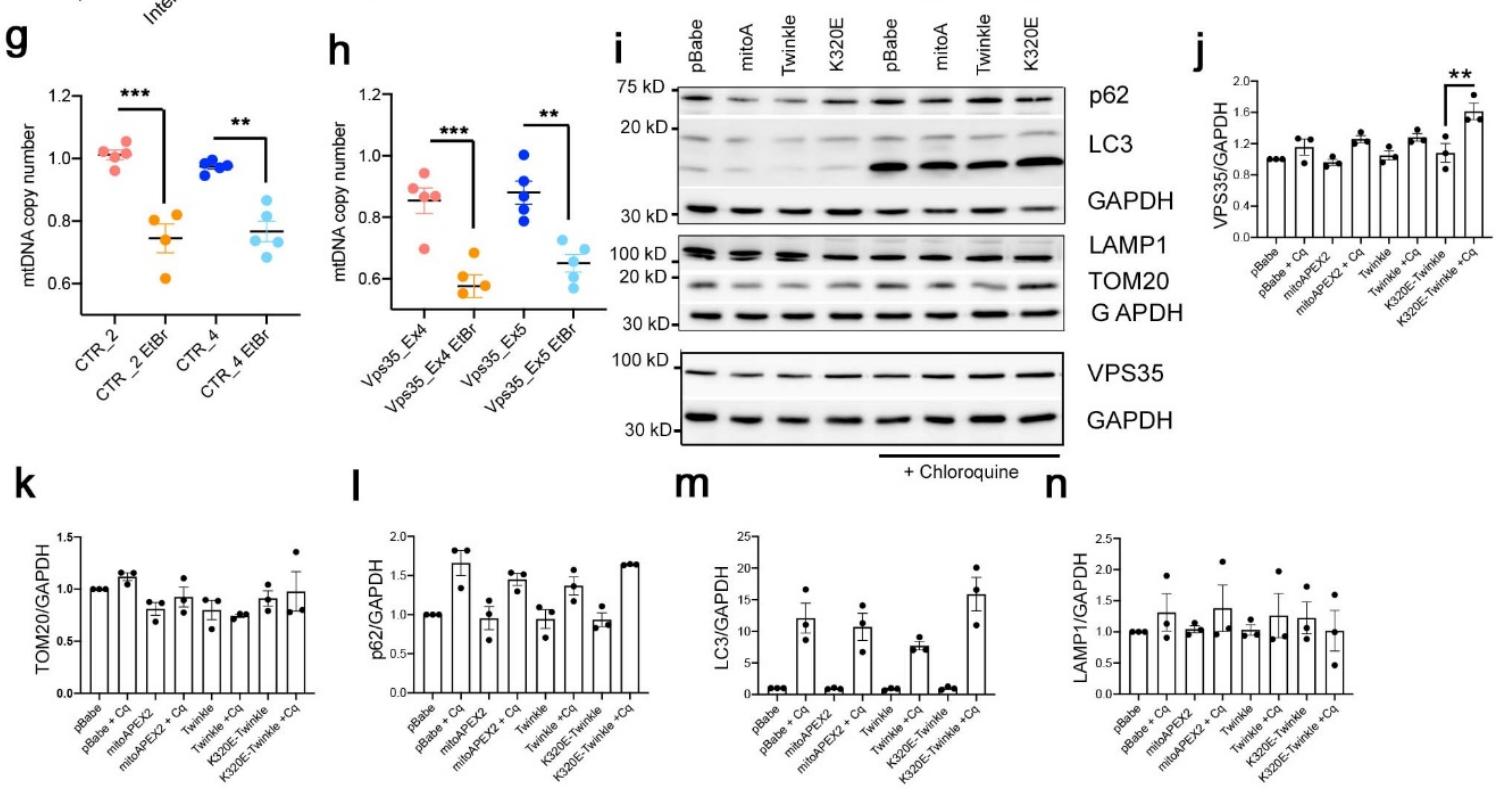

I
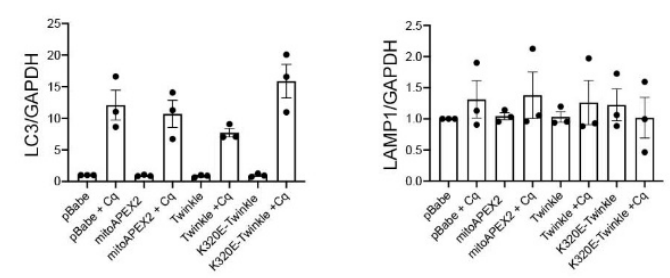

Figure S7. (a) mtDNA copy number in $\mathrm{C} 2 \mathrm{C} 12$ cells treated for 7 days with $50 \mathrm{ng} / \mathrm{ml} \mathrm{EtBr.} \mathrm{n}=3$. (b) Western blot analysis of MEFs after CRISPR Cas9 mediated KO of Vps35. (c) Schematic representation of DNA modification in Vps35 Exon 4 and Exon 5 triggered by CRISPR-Cas9. (d) Quantification of mitochondrial morphology in Vps35 KO clones. $\mathrm{n}=3,>30$ cells per replicate. (e) mtDNA foci quantification in control and (f) Vps35 KO clones treated with $\mathrm{EtBr}$. $\mathrm{n}=3$. (g) qPCR mtDNA copy number analysis of control and (h) Vps35 KO clones treated with EtBr. Students' T-test. ${ }^{* *}, p<0.01 ;{ }^{* * *}, p<0.001 . n=5$. (i) Western blot analysis of autophagy markers and VPS35 levels in C2C12 cells expressing Twinkle-APEX2-V5 variants (WT, $\mathrm{KE}$ ) or matrix targeted APEX2 protein, treated $24 \mathrm{~h}$ with $10 \mu \mathrm{M}$ Chloroquine. $n=3$. (j-n) Intensity quantification of the indicated proteins relative to empty vector $\mathrm{pB}$ (pBABE). (a, $\mathbf{g}, \mathbf{h}$ and $\mathbf{j}-\mathbf{n})$ ANOVA analysis of variance. Tukey multiple comparison test. Mean \pm SEM. *,$p<0.05 ;{ }^{* *}, p<0.01$; ${ }^{* * *}, p<0.001$. (d) Two-way ANOVA. Control lines were compared with KO lines. ${ }^{* *}, p<0.01$; $* * * *, p<0.0001$. 

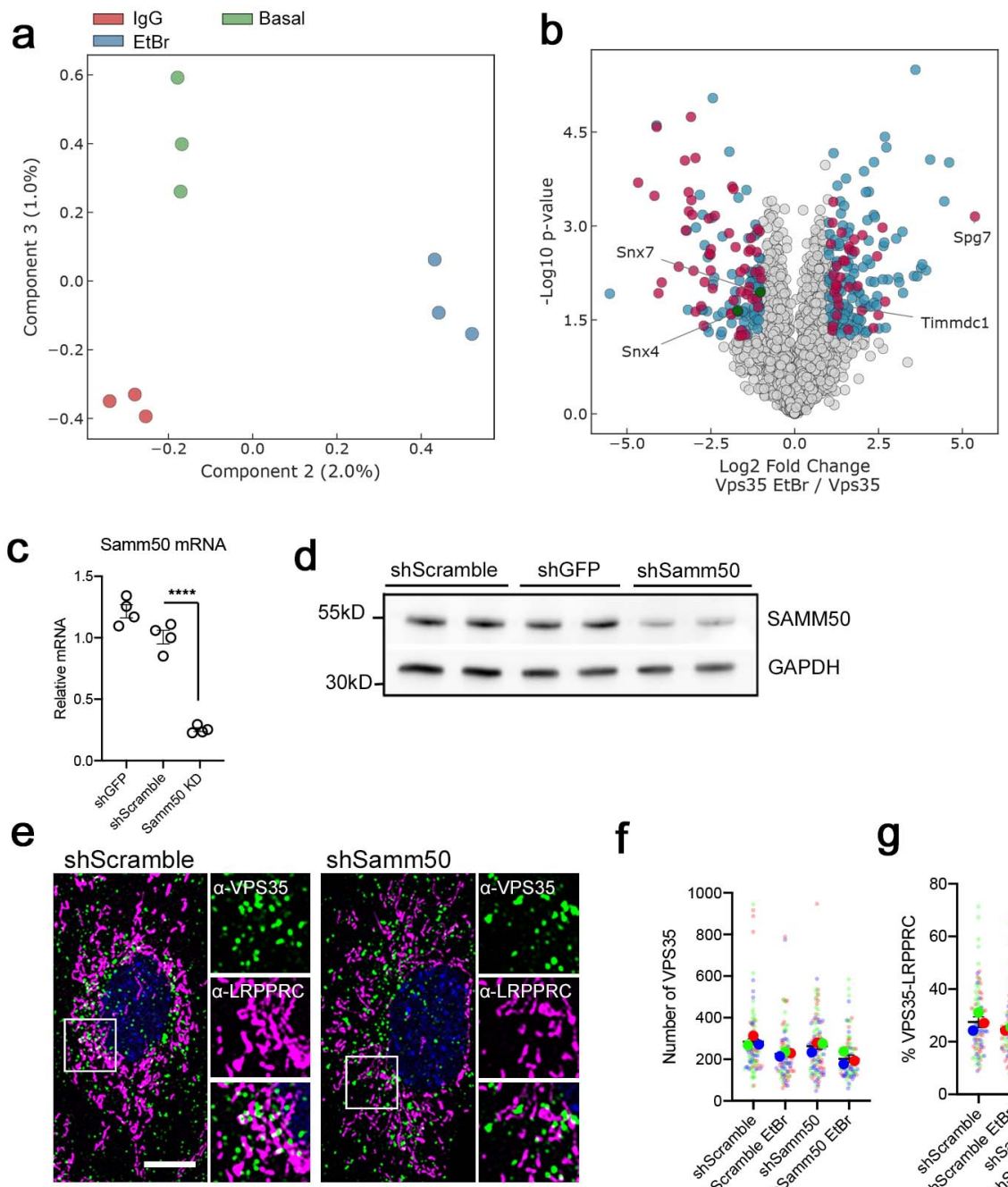

g
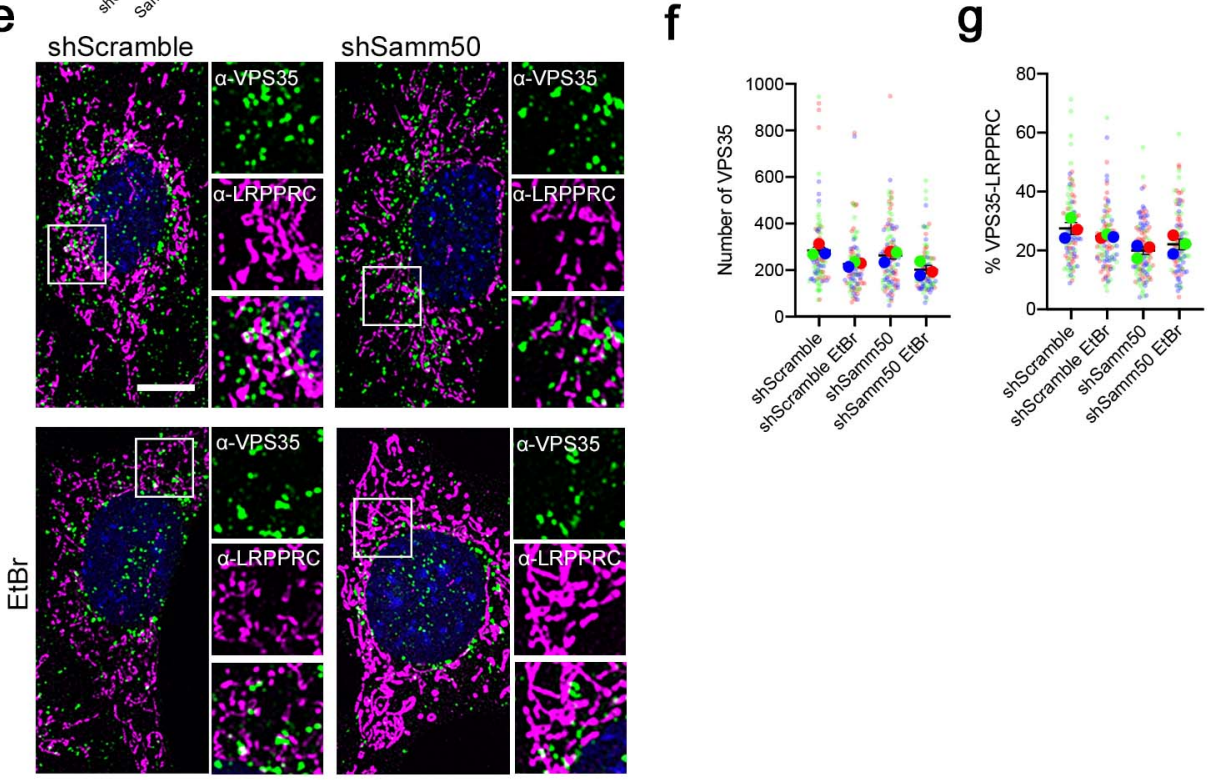

Figure S8. (a) Component analysis of VPS35 IP. (b) Comparison of VPS35 IP profiles from cells in basal medium or treated with $50 \mathrm{ng} / \mathrm{ml} \mathrm{EtBr}$ for 1 week. Only significantly different proteins are highlighted. Blue, cytosolic proteins; Red, mitochondrial proteins; Green, retromer proteins. (c) mRNA quantification and (d) western blot analysis of Samm50 upon shRNA transduction. Gapdh mRNA and GAPDH was used as an internal control for both experiments. (e) Immunofluorescence of Control and Samm50 KD MEFs labelled with a-VPS35 and a-LRPPRC in basal and 7 days treated with 50ng/ml EtBr. Scale bar, 10 $\mu \mathrm{m}$. (f, $\mathbf{g})$ Quantification of VPS35 particles and VPS35 in contact with LRPPRC. $n=3$. Mean \pm SEM. ANOVA analysis of variance. Tukey multiple comparison test. 
a

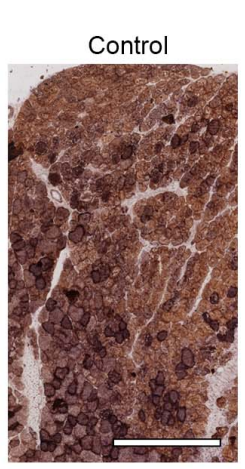

d

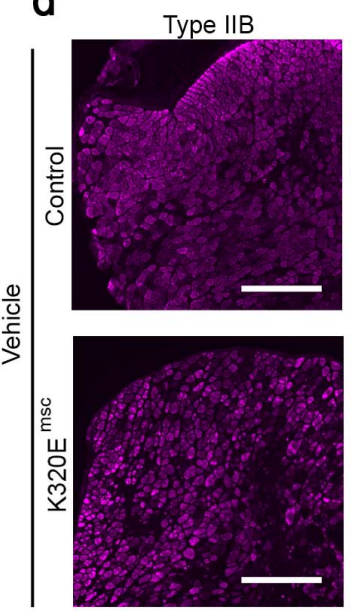

e

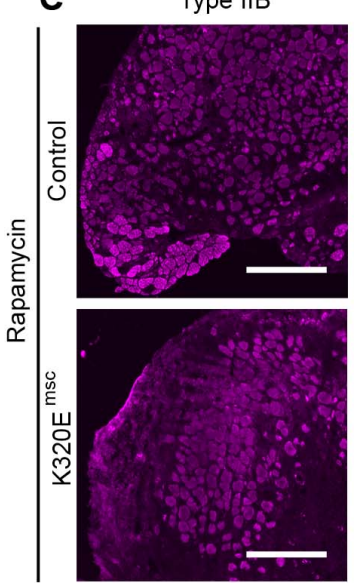

b

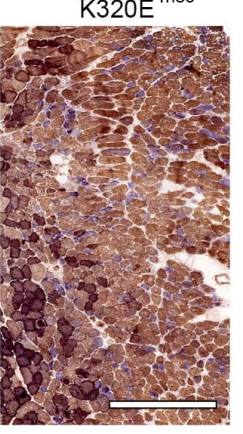

b

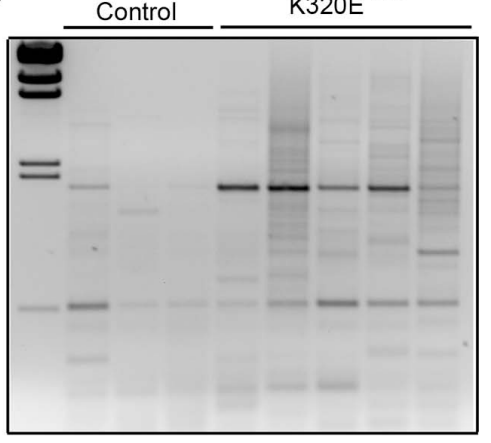

C

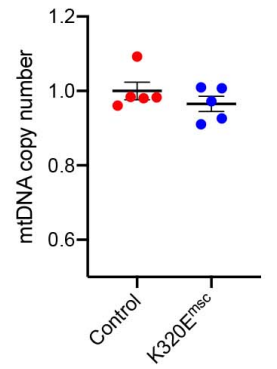

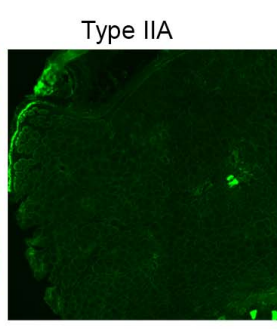
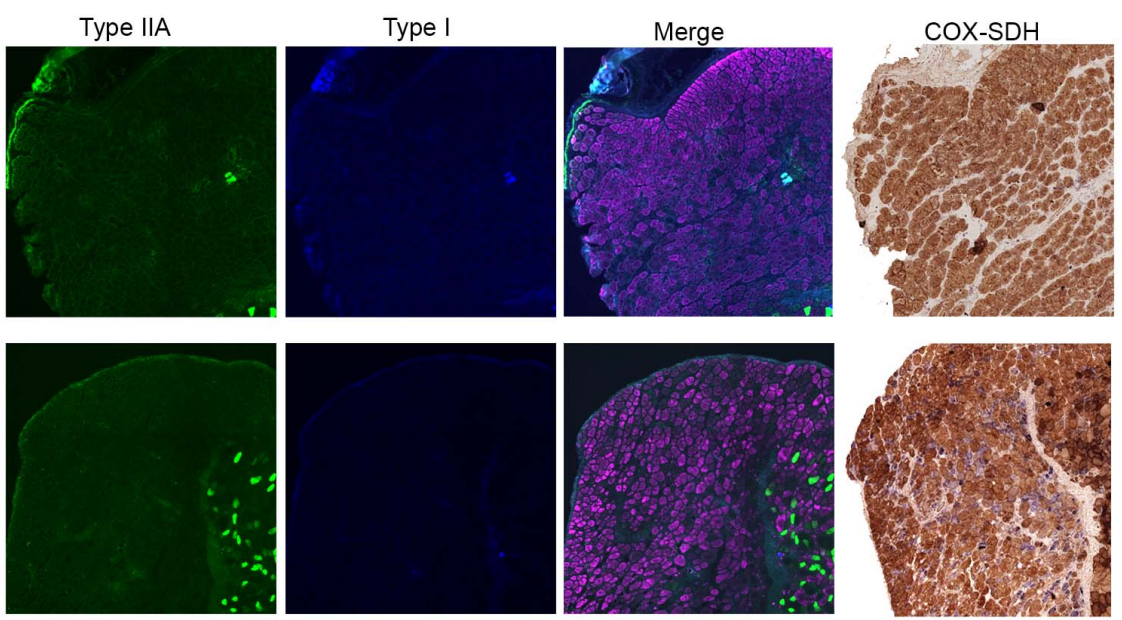

Type IIA

Type I

Merge

COX-SDH
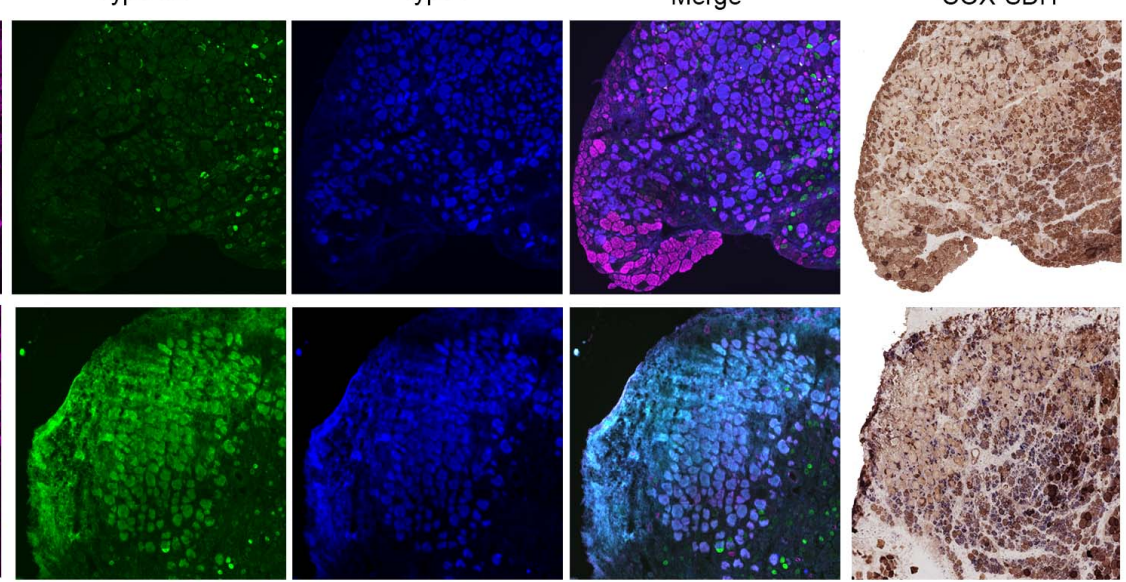

Figure S9. (a) Representative image for COX-SDH staining in a regenerated area from the M. Tibialis anterior from control and K320E-Twinkle ${ }^{\mathrm{msc}}$-mice. Bar, $500 \mu \mathrm{m}$. (b) Long Range PCR for mtDNA deletions and, (c) mtDNA copy number analysis in control and Twinkle-K320E mice. $n=5$. Muscle regeneration was induced with intramuscular injection of 10 $\mu \mathrm{M}$ Cardiotoxin (Naja Pallida). Students' T-test. (d) Fiber type and COX-SDH serial analysis of regenerated muscles in vehicle and (e) rapamycin treated mice. Mean \pm SEM. Bar, $250 \mu \mathrm{m}$. 


\section{Supplementary Files}

This is a list of supplementary files associated with this preprint. Click to download.

- SupTable1.xlsx

- SupTable2.xIsx

- SupTable3.xIsx 